\title{
Solvability for a New Class of Moore-Gibson-Thompson Equation with Viscoelastic Memory, Source Terms, and Integral Condition
}

\author{
Salah Mahmoud Boulaaras $\mathbb{D}^{1,2}$ Abdelbaki Choucha, ${ }^{3}$ Djamel Ouchenane, ${ }^{4}$ Asma Alharbi, ${ }^{1}$ \\ Mohamed Abdalla $₫{ }^{5,6}$ and Bahri Belkacem Cherif $\oplus^{1,7}$ \\ ${ }^{1}$ Department of Mathematics, College of Sciences and Arts, ArRas, Qassim University, Saudi Arabia \\ ${ }^{2}$ Laboratory of Fundamental and Applied Mathematics of Oran (LMFAO), University of Oran 1, Oran, 31000 Oran, Algeria \\ ${ }^{3}$ Laboratory of Operator Theory and PDEs: Foundations and Applications, Department of Mathematics, Faculty of Exact Sciences, \\ University of El Oued, Algeria \\ ${ }^{4}$ Laboratory of Pure and Applied Mathematics, Amar Teledji Laghouat University, Algeria \\ ${ }^{5}$ Mathematics Department, College of Science, King Khalid University, Abha 61413, Saudi Arabia \\ ${ }^{6}$ Mathematics Department, Faculty of Science, South Valley University, Qena 83523, Egypt \\ ${ }^{7}$ Preparatory Institute for Engineering Studies in Sfax, Tunisia
}

Correspondence should be addressed to Bahri Belkacem Cherif; bahi1968@yahoo.com

Received 23 March 2021; Accepted 1 April 2021; Published 20 April 2021

Academic Editor: Santosh Kumar

Copyright () 2021 Salah Mahmoud Boulaaras et al. This is an open access article distributed under the Creative Commons Attribution License, which permits unrestricted use, distribution, and reproduction in any medium, provided the original work is properly cited.

This paper deals with the existence and uniqueness of solutions of a new class of Moore-Gibson-Thompson equation with respect to the nonlocal mixed boundary value problem, source term, and nonnegative memory kernel. Galerkin's method was the main used tool for proving our result. This work is a generalization of recent homogenous work.

\section{Introduction}

In this contribution, we are interested to study the existence and uniqueness of solutions of the following problem

$$
\left\{\begin{array}{l}
\mathscr{L} u(x, t)=a u_{t t t}+\beta u_{t t}-c^{2} \Delta u-b \Delta u_{t}-\int_{0}^{t} h(t-\sigma) \Delta u(\sigma) d \sigma=F(x, t), \\
u(x, 0)=u_{0}(x), u_{t}(x, 0)=u_{1}(x), u_{t t}(x, 0)=u_{2}(x) \\
\frac{\partial u}{\partial \eta}=\int_{0}^{t} \int_{\Omega}(\xi, \tau) d \xi d \tau, x \in \partial \Omega .
\end{array}\right.
$$

Here, $a$ and $\beta$ are physical parameters, and $c$ is the speed of sound. The convolution term $\int_{0}^{t} h(t-s) \Delta u(s) d s$ reflects the memory effect of materials due to vicoelasticity, $F$ is a given function, and $h$ is the relaxation function satisfying
(H1) $h \in C^{1}(\mathbb{R}+, \mathbb{R}+)$ is a nonincreasing function satisfying

$$
h(0)>0,1-h_{0}=l>0,
$$

where $\quad h_{0}=G(\infty)=\int_{0}^{\infty} h(s) d s>0, \quad G(t)=\int_{0}^{t} h(s) d s, \quad$ and $h^{\prime \prime}>0$.

(H2) $\exists \zeta>0$ satisfying

$$
h^{\prime}(t) \leq-\zeta h(t), t \geq 0 \text {. }
$$

$$
\beta-a>0
$$


The phenomena resulting from sound waves (diffraction, interference, reflection) in terms of modeling are very important. As the existence of the third derivative is very important, especially in the field of thermodynamics (EIT), the study of these models is considered the beginning of an in-depth understanding of both convergent and good behavior. From the results extracted, the equation of MGT resulted in nonlinear acoustics, for much depth, see ([1-7]) and especially [8] where equation of MGT appeared for the first time. Also, nonlinear problems of great importance can be considered [9], where Galerkin's method was applied in solving them, for more depth $([2,3,10-13])$. Recently, in [14], the authors studied the equation of MGT with memory. Likewise, in [1], the authors used Galerkin's method to demonstrate the ability to solve a mixed problem of MGT equation in the absence of viscous elasticity and memory. Based on work [9] and the works we mentioned earlier, we want to prove the existence and uniqueness of a weak solution to the problem (1).

We divide this paper into the following: in the second part, we put some definitions and appropriate spaces. Then, we apply Galerkin's method to prove the existence, and in the fourth part, we demonstrate the uniqueness.

\section{Preliminaries}

We will define the spaces: $V\left(Q_{T}\right)$ and $W\left(Q_{T}\right)$ by

$$
\begin{aligned}
V\left(Q_{T}\right) & =\left\{u \in W_{2}^{1}\left(Q_{T}\right): u_{t} \in W_{2}^{1}\left(Q_{T}\right), u, \nabla u \in L_{h}^{2}\left(Q_{T}\right)\right\}, \\
W\left(Q_{T}\right) & =\left\{u \in V\left(Q_{T}\right): u(x, T)=0\right\}, \\
L_{h}^{2}\left(Q_{T}\right) & =\left\{u \in V\left(Q_{T}\right): \int_{0}^{T} h \circ u(t) d t<\infty\right\},
\end{aligned}
$$

where

$$
h \circ u(t)=\int_{\Omega} \int_{0}^{t} h(t-\sigma)(u(t)-u(\sigma))^{2} d \sigma d x .
$$

Consider the equation

$$
\begin{gathered}
a\left(u_{t t t}, v\right)_{L^{2}\left(Q_{T}\right)}+\beta\left(u_{t t}, v\right)_{L^{2}\left(Q_{T}\right)}-c^{2}(\Delta u, v)_{L^{2}\left(Q_{T}\right)} \\
-b\left(\Delta u_{t}, v\right)_{L^{2}\left(Q_{T}\right)}-(\Delta w, v)_{L^{2}\left(Q_{T}\right)}=(F, v)_{L^{2}\left(Q_{T}\right)},
\end{gathered}
$$

where

$$
w(x, t)=\int_{0}^{t} h(t-\sigma) u(x, \sigma) d \sigma
$$

and (., . $)_{L 2(Q T)}$ stands for the inner product in $L^{2}\left(Q_{T}\right), u$ is supposed to be a solution of (1) and $v \in W\left(Q_{T}\right)$. Evaluation of the inner product in [9] gives

$$
\begin{aligned}
-a\left(u_{t t}, v_{t}\right)_{L^{2}\left(Q_{T}\right)}-\beta\left(u_{t}, v_{t}\right)_{L^{2}\left(Q_{T}\right)}+c^{2}(\nabla u, \nabla v)_{L^{2}\left(Q_{T}\right)} & +b\left(\nabla u_{t}, \nabla v\right)_{L^{2}\left(Q_{T}\right)}+(\nabla w, \nabla v)_{L^{2}\left(Q_{T}\right)} \\
= & (F, v)_{L^{2}\left(Q_{T}\right)}+c^{2} \int_{0}^{T} \int_{\partial \Omega} v\left(\int_{0}^{t} \int_{\Omega} v(\xi, \tau) d \xi d \tau\right) d s_{x} d t \\
& +b \int_{0}^{T} \int_{\partial \Omega} v \int_{\Omega} u(\xi, t) d \xi d s_{x} d t-b \int_{0}^{T} \int_{\partial \Omega} v \int_{\Omega} u_{0}(\xi) d \xi d s_{x} d t \\
& +a\left(u_{2}(x), v(x, 0)\right)_{L^{2}(\Omega)}+\beta\left(u_{1}(x), v(x, 0)\right)_{L^{2}(\Omega)} \\
& +\int_{0}^{T} \int_{\partial \Omega} v\left(\int_{0}^{t} \int_{\Omega} w(\xi, \tau) d \xi d \tau\right) d s_{x} d t .
\end{aligned}
$$

We give two useful inequalities:

(i) Gronwall inequality. Let the nonnegative integrable functions $\varphi(t), \phi(t)$ on the interval $I$ with the nondecreasing function $h(t)$. If $\forall t \in I$, we have

$$
\phi(t) \leq \varphi(t)+c \int_{0}^{t} \phi(s) d s
$$

where $c>0$, hence,

$$
\phi(\mathrm{t}) \leq \varphi(\mathrm{t}) \exp (\mathrm{ct})
$$

(ii) Trace inequality (see [15]). If $\Phi \in W_{1}^{2}(\Omega)$ where $\Omega$ is a bounded domain in $\mathbb{R}^{n}$ with smooth boundary $\partial \Omega$, then for any $\varepsilon>0$,

$$
\|\Phi\|_{L^{2}(\partial \Omega)}^{2} \leq \varepsilon\|\nabla \Phi\|_{L^{2}(\Omega)}^{2}+l(\varepsilon)\|\Phi\|_{L^{2}(\Omega)}^{2},
$$

where $l(\varepsilon)>0$.

Definition 1. We call a generalized solution to the problem (1) for each function $u \in V\left(Q_{T}\right)$ that fulfills the equation (9) for each $v \in W\left(Q_{T}\right)$.

\section{Solvability of the Problem}

In this section, we use Galerkin's method to prove the existence of a generalized solution of our problem.

Theorem 2. If $u_{0} \in W_{2}^{1}(\Omega), u_{1} \in W_{2}^{1}(\Omega), u_{2} \in L^{2}(\Omega)$, and $F \in L^{2}\left(Q_{T}\right)$, then there is at least one generalized solution in $V\left(Q_{T}\right)$ to problem (1).

Proof. Let $\left\{Z_{k}(x)\right\}_{k \geq 1}$ be a fundamental system in $W_{2}^{1}(\Omega)$, such that $\left(Z_{k}, Z_{l}\right) \Omega=\delta_{k, l}$. 
First, we will give an approximate solution of the problem (1) in the form

$$
u^{N}(x, t)=\sum_{k=1}^{N} C_{k}(t) Z_{k}(x),
$$

where $C_{k}(t)$ are constants given by the conditions, for $k=$ $1, \cdots, N$,

$$
\left(\mathscr{L} u(x, t), Z_{l}(x)\right)_{L^{2}(\Omega)}=\left(F(x, t), Z_{l}(x)\right)_{L^{2}(\Omega)}
$$

and can be determined from the relations

$$
\begin{aligned}
a\left(u_{t t t}^{N},\right. & \left.Z_{l}(x)\right)_{L^{2}(\Omega)}+\beta\left(u_{t t}^{N}, Z_{l}(x)\right)_{L^{2}(\Omega)}+c^{2}\left(\nabla u^{N}, \nabla Z_{l}(x)\right)_{L^{2}(\Omega)} \\
& +b\left(\nabla u_{t}^{N}, \nabla Z_{l}(x)\right)_{L^{2}(\Omega)}+\left(\nabla w^{N}, \nabla Z_{l}(x)\right)_{L^{2}(\Omega)} \\
= & \left(F(x, t), Z_{l}(x)\right)_{L^{2}(\Omega)}+c^{2} \int_{\partial \Omega} Z_{l}(x)\left(\int_{0}^{t} \int_{\Omega} u^{N}(\xi, \tau) d \xi d \tau\right) d s_{x} \\
& +b \int_{\partial \Omega} Z_{l}(x)\left(\int_{0}^{t} \int_{\Omega} u^{N}(\xi, \tau) d \xi d \tau\right) d s_{x} \\
& +\int_{\partial \Omega} Z_{l}(x)\left(\int_{0}^{t} \int_{\Omega} w^{N}(\xi, \tau) d \xi d \tau\right) d s_{x},
\end{aligned}
$$

substitution of (13) into (15), and we find for $l=1, \cdots, N$.

$$
\begin{aligned}
\int_{\Omega} \sum_{k=1}^{N} & \left\{a C_{k}^{\prime \prime \prime}(t) Z_{k}(x) Z_{l}(x) \beta C_{k}^{\prime \prime \prime}(t) Z_{k}(x) Z_{l}(x)\right. \\
& +c^{2} C_{k}(t) \nabla Z_{k}(x) \cdot \nabla Z_{l}(x)+b C_{k}^{\prime}(t) \nabla Z_{k}(x) \cdot \nabla Z_{l}(x) \\
& \left.+\left(\int_{0}^{t} h(t-\sigma) C_{k}(\sigma) d \sigma\right) \nabla Z_{k}(x) \cdot \nabla Z_{l}(x)\right\} d x \\
= & \left(F(x, t), Z_{l}(x)\right)_{L^{2}(\Omega)}+c^{2} \sum_{k=1}^{N} \int_{0}^{t} C_{k}(\tau)\left(\int_{\partial \Omega} Z_{l}(x) \int_{\Omega} Z_{k}(\xi) d \xi d s_{x}\right) d \tau \\
& +b \sum_{k=1}^{N} \int_{0}^{t} C_{k}^{\prime}(\tau)\left(\int_{\partial \Omega} Z_{l}(x) \int_{\Omega} Z_{k}(\xi) d \xi d s_{x}\right) d \tau \\
& +\sum_{k=1}^{N} \int_{0}^{t}\left(\int_{0}^{\tau} h(\tau-\sigma) C_{k}(\sigma) d \sigma\right)\left(\int_{\partial \Omega} Z_{l}(x) \int_{\Omega} Z_{k}(\xi) d \xi d s_{x}\right) d \tau
\end{aligned}
$$

From (15) it follows that

$$
\begin{aligned}
\sum_{k=1}^{N}\{a & C_{k}^{\prime \prime \prime}(t)\left(Z_{k}(x), Z_{l}(x)\right)_{L^{2}(\Omega)}+\beta C_{k}^{\prime \prime}(t)\left(Z_{k}(x), Z_{l}(x)\right)_{L^{2}(\Omega)} \\
& +c^{2} C_{k}(t)\left(\nabla Z_{k}(x), \nabla Z_{l}(x)\right)_{L^{2}(\Omega)} \\
& +b C_{k}^{\prime}(t)\left(\nabla Z_{k}(x), \nabla Z_{l}(x)\right)_{L^{2}(\Omega)} \\
& \left.+\left(\int_{0}^{t} h(t-\sigma) C_{k}(\sigma) d \sigma\right)\left(\nabla Z_{k}(x), \nabla Z_{l}(x)\right)_{L^{2}(\Omega)}\right\} \\
= & \left(F(x, t), Z_{l}(x)\right)_{L^{2}(\Omega)}+c^{2} \sum_{k=1}^{N} \int_{0}^{t} C_{k}(\tau)\left(\int_{\partial \Omega} Z_{l}(x) \int_{\Omega} Z_{k}(\xi) d \xi d s_{x}\right) d \tau \\
& +b \sum_{k=1}^{N} \int_{0}^{t} C_{k}^{\prime}(\tau)\left(\int_{\partial \Omega} Z_{l}(x) \int_{\Omega} Z_{k}(\xi) d \xi d s_{x}\right) d \tau \\
& +\sum_{k=1}^{N} \int_{0}^{t}\left(\int_{0}^{\tau} h(\tau-\sigma) C_{k}(\sigma) d \sigma\right)\left(\int_{\partial \Omega} Z_{l}(x) \int_{\Omega} Z_{k}(\xi) d \xi d s_{x}\right) d \tau
\end{aligned}
$$

Let

$$
\begin{aligned}
\left(Z_{k}, Z_{l}\right)_{L^{2}(\Omega)} & =\delta_{k l}=\left\{\begin{array}{cc}
1, & k=l \\
0, & k \neq l
\end{array},\right. \\
\left(\nabla Z_{k}, \nabla Z_{l}\right)_{L^{2}(\Omega)} & =\gamma_{k l}, \\
\int_{\partial \Omega} Z_{l}(x) \int_{\Omega} Z_{k}(\xi) d \xi d s & =\chi_{k l}, \\
\left(F(x, t), Z_{l}(x)\right)_{L^{2}(\Omega)} & =F_{l}(t) .
\end{aligned}
$$

Then, (17) can be written as

$$
\begin{aligned}
& \sum_{k=1}^{N}\left\{a \delta_{k l} C_{k}^{\prime \prime \prime}(t)+\beta \delta_{k l} C_{k}^{\prime \prime}(t)+c^{2} C_{k}(t) \gamma_{k l}\right. \\
& \quad+\left(\int_{0}^{t} h(t-\sigma) C_{k}(\sigma) d \sigma\right) \gamma_{k l}+b C_{k}^{\prime}(t) \gamma_{k l} \\
& \left.\quad-\int_{0}^{t}\left[c^{2} C_{k}(\tau) \chi_{k l}-\left(\int_{0}^{\tau} h(\tau-\sigma) C_{k}(\sigma) d \sigma\right) \chi_{k l}\right] d \tau\right\}=F_{l}(t) .
\end{aligned}
$$

By differentiating (two times) with respect to $t$, it gives

$$
\begin{gathered}
\sum_{k=1}^{N}\left\{a \delta_{k l} C_{k}^{\prime \prime \prime}(t)+\beta \delta_{k l} C_{k}^{\prime \prime \prime}(t)+c^{2} C_{k}^{\prime \prime}(t) \gamma_{k l}+h(0) C_{k}^{\prime}(t) \gamma_{k l}\right. \\
\left.+b C_{k}^{\prime \prime}(t) \gamma_{k l}-c^{2} C_{k}(t) \chi_{k l}-b C_{k}^{\prime \prime}(t) \chi_{k l}-h(0) C_{k}(t) \chi_{k l}\right\}=F_{l}^{\prime \prime}(t) . \\
\sum_{k=1}^{N}\left\{a \delta_{k l} C_{k}^{\prime \prime \prime}(0)+\beta \delta_{k l} C_{k}^{\prime \prime}\right\} .
\end{gathered}
$$

We find a system of differential equations of fifth order with respect to $t$, constant coefficients, and the initial conditions (21). Hence, we obtain a Cauchy problem of linear differential equations with smooth coefficients that is uniquely solvable. Thus, $\forall n, \exists u^{N}(x)$ satisfying (15).

Now, we prove that $u^{N}$ is sequence bounded. To do this, we multiply each equation of (15) by the appropriate $C_{k}^{\prime}(t)$ summing over $k$ from 1 to $N$. Hence, by integration the result equality with respect to $t$ from 0 to $\tau$, and $\tau \leq T$, it gives

$$
\begin{aligned}
& a\left(u_{t t t}^{N}, u_{t}^{N}\right)_{L^{2}\left(Q_{\tau}\right)}+\beta\left(u_{t t t}^{N}, u_{t}^{N}\right)_{L^{2}\left(Q_{\tau}\right)}+c^{2}\left(\nabla u^{N}, \nabla u_{t}^{N}\right)_{L^{2}\left(Q_{\tau}\right)} \\
& \quad+b\left(\nabla u_{t}^{N}, \nabla u_{t}^{N}\right)_{L^{2}\left(Q_{\tau}\right)}+\left(\nabla w^{N}, \nabla u_{t}^{N}\right)_{L^{2}\left(Q_{\tau}\right)} \\
& =\left(F, u_{t}^{N}\right)_{L^{2}\left(Q_{\tau}\right)}+c^{2} \int_{0}^{\tau} \int_{0}^{\tau} u_{t}^{N}(x, t)\left(\int_{0}^{t} \int_{\Omega}(\xi, \eta) d \xi d \eta\right) d s_{x} d t \\
& \quad+b \int_{0}^{\tau} \int_{\partial \Omega} u_{t}^{N}(x, t)\left(\int_{0}^{t} \int_{\Omega} u_{t}^{N}(\xi, \eta) d \xi d \eta\right) d s_{x} d t \\
& \quad+\int_{0}^{\tau} \int_{\partial \Omega} u_{t}^{N}(x, t)\left(\int_{0}^{t} \int_{\Omega} w^{N}(\xi, \eta) d \xi d \eta\right) d s_{x} d t
\end{aligned}
$$


Evaluation of the terms on the LHS of (22) gives

$$
\begin{aligned}
a\left(u_{t t t}^{N}, u_{t}^{N}\right)_{L^{2}\left(Q_{\tau}\right)}= & a\left(u_{\tau \tau}^{N}, u_{\tau}^{N}\right)_{L^{2}(\Omega)}-a\left(u_{t t}^{N}(x, 0), u_{t}^{N}(x, 0)\right)_{L^{2}(\Omega)} \\
& -a \int_{0}^{\tau}\left\|u_{t t}(x, t)\right\|_{L^{2}(\Omega)}^{2} d t
\end{aligned}
$$

$$
\beta\left(u_{t t}^{N}, u_{t}^{N}\right)_{L^{2}\left(Q_{\tau}\right)}=\frac{\beta}{2}\left\|u_{\tau}^{N}(x, \tau)\right\|_{L^{2}(\Omega)}^{2}-\frac{\beta}{2}\left\|u_{t}^{N}(x, 0)\right\|_{L^{2}(\Omega)}^{2}
$$

$$
\begin{aligned}
c^{2}\left(\nabla u^{N}, \nabla u_{t}^{N}\right)_{L^{2}\left(Q_{\tau}\right)}= & \frac{c^{2}}{c}\left\|\nabla u^{N}(x, \tau)\right\|_{L^{2}(\Omega)}^{2} \\
& -\frac{c^{2}}{c}\left\|\nabla u^{N}(x, 0)\right\|_{L^{2}(\Omega)}^{2},
\end{aligned}
$$

$$
b\left(\nabla u_{t}^{N}, \nabla u_{t}^{N}\right)_{L^{2}\left(Q_{\tau}\right)}=b \int_{0}^{\tau}\left\|\nabla u_{t}^{N}(x, t)\right\|_{L^{2}(\Omega)}^{2} d t
$$

$\left(\nabla w^{N}, \nabla u_{t}^{N}\right)_{L^{2}\left(Q_{\tau}\right)}=\frac{1}{2} h \circ \nabla u^{N}(\tau)-\frac{1}{2} G(\tau)\left\|\nabla u^{N}(x, \tau)\right\|_{L^{2}(\Omega)}^{2}$

$$
\begin{aligned}
& -\frac{1}{2} \int_{0}^{\tau} h^{\prime N}(t) d t \\
& +\frac{1}{2} \int_{0}^{\tau} h(t)\left\|\nabla u^{N}(x, \tau)\right\|_{L^{2}(\Omega)}^{2} d t,
\end{aligned}
$$

$$
\begin{aligned}
c^{2} \int_{0}^{\tau} \int_{\partial \Omega} u_{t}^{N}\left(\int_{0}^{t} \int_{\Omega} u^{N}(\xi, \eta) d \xi d \eta\right) d s_{x} d t \\
=c^{2} \int_{\partial \Omega} u^{N}(x, \tau) \int_{0}^{\tau} \int_{\Omega} u^{N}(\xi, t) d \xi d t d s_{x} \\
\quad-c^{2} \int_{\partial \Omega} \int_{0}^{\tau} u^{N}(x, t) \int_{\Omega} u^{N}(\xi, t) d \xi d t d s_{x}
\end{aligned}
$$

So,

$$
\begin{aligned}
b \int_{0}^{\tau} \int_{\partial \Omega} u_{t}^{N}\left(\int_{0}^{t} \int_{\Omega} u_{t}^{N}(\xi, \eta) d \xi d \eta\right) d s_{x} d t \\
=b \int_{\partial \Omega} \int_{0}^{\tau} u_{t}^{N}(x, t) \int_{\Omega} u^{N}(\xi, t) d \xi d t d s_{x} \\
\quad-b \int_{\partial \Omega} \int_{0}^{\tau} u_{t}^{N}(x, t) \int_{\Omega} u^{N}(\xi, 0) d \xi d t d s_{x} .
\end{aligned}
$$

Thus,

$$
\begin{gathered}
\int_{0}^{\tau} \int_{\partial \Omega} u_{t}^{N}\left(\int_{0}^{t} \int_{\Omega} w^{N}(\xi, \eta) d \xi d \eta\right) d s_{x} d t \\
=\int_{\partial \Omega} u^{N}(x, \tau) \int_{0}^{\tau} \int_{\Omega} u^{N}(\xi, t) d \xi d t d s_{x} \\
\quad-\int_{\partial \Omega} \int_{0}^{\tau} u^{N}(x, t) \int_{\Omega} w^{N}(\xi, t) d \xi d t d s_{x} .
\end{gathered}
$$

Taking into account the equalities (23)-(30) in (22), we end up with

$$
\begin{aligned}
& a\left(u_{\tau \tau}^{N}, u_{\tau}^{N}\right)_{L^{2}(\Omega)}+\frac{\beta}{2}\left\|u_{\tau}^{N}(x, \tau)\right\|_{L^{2}(\Omega)}^{2} \\
& +\frac{c^{2}}{2}\left\|\nabla u^{N}(x, \tau)\right\|_{L^{2}(\Omega)}^{2} \frac{1}{2} h \circ \nabla u^{N}(\tau) \\
& -\frac{1}{2} G(\tau)\left\|\nabla u^{N}(x, \tau)\right\|_{L^{2}(\Omega)}^{2} \\
& =a\left(u_{t t}^{N}(x, 0), u_{t}^{N}(x, 0)\right)_{L^{2}(\Omega)}+\frac{\beta}{2}\left\|u_{\tau}^{N}(x, 0)\right\|_{L^{2}(\Omega)}^{2} \\
& +\frac{c^{2}}{2}\left\|\nabla u^{N}(x, 0)\right\|_{L^{2}(\Omega)}^{2}+a \int_{0}^{\tau}\left\|u_{t t}(x, t)\right\|_{L^{2}(\Omega)}^{2} d t \\
& +b \int_{0}^{\tau}\left\|\nabla u_{t}^{N}(x, t)\right\|_{L^{2}(\Omega)}^{2} d t+\frac{1}{2} \int_{0}^{\tau} h^{\prime N}(t) d t \\
& -\frac{1}{2} \int_{0}^{\tau} h(t)\left\|\nabla u^{N}(x, t)\right\|_{L^{2}(\Omega)}^{2} d t \\
& +c^{2} \int_{\partial \Omega} u^{N}(x, \tau) \int_{0}^{\tau} \int_{\Omega} u^{N}(\xi, t) d \xi d t d s_{x} \\
& -c^{2} \int_{\partial \Omega} \int_{0}^{\tau} u^{N}(x, t) \int_{\Omega}(\xi, t) d \xi d t d s_{x} \\
& +b \int_{\partial \Omega} \int_{0}^{\tau} u_{t}^{N}(x, t) \int_{\Omega} u^{N}(\xi, t) d \xi d t d s_{x} \\
& -b \int_{\partial \Omega} \int_{0}^{\tau} u_{t}^{N}(x, t) \int_{\Omega} u^{N}(\xi, 0) d \xi d t d s_{x} \\
& +\int_{\partial \Omega} u^{N}(x, \tau) \int_{0}^{\tau} \int_{\Omega}(\xi, t) d \xi d t d s_{x} \\
& -\int_{\partial \Omega} \int_{0}^{\tau} u^{N}(x, t) \int_{\Omega} w^{N}(\xi, t) d \xi d t d s_{x}+\left(F, u_{t}^{N}\right)_{L^{2}\left(Q_{\tau}\right)}
\end{aligned}
$$

Now, multiplying the equations of (15) by $C_{k}^{\prime \prime}(t)$, collect them from 1 to $N$ and integrating the result with respect to $t$ from 0 to $\tau$, and $\tau \leq T$, we find

$$
\begin{aligned}
& a\left(u_{t t t}^{N}, u_{t t}^{N}\right)_{L^{2}\left(Q_{\tau}\right)}+\beta\left(u_{t t}^{N}, u_{t t}^{N}\right)_{L^{2}\left(Q_{\tau}\right)}+c^{2}\left(\nabla u^{N}, \nabla u_{t t}^{N}\right)_{L^{2}\left(Q_{\tau}\right)} \\
& \quad+b\left(\nabla u_{t}^{N}, \nabla u_{t t}^{N}\right)_{L^{2}\left(Q_{\tau}\right)}+\left(\nabla w^{N}, \nabla u_{t t}^{N}\right)_{L^{2}\left(Q_{\tau}\right)} \\
& =\left(F, u_{t t}^{N}\right)_{L^{2}\left(Q_{\tau}\right)}+c^{2} \int_{0}^{\tau} \int_{\partial \Omega} u_{t t}^{N}(x, t)\left(\int_{0}^{t} \int_{\Omega} u^{N}(\xi, \eta) d \xi d \eta\right) d s_{x} d t \\
& \quad+b \int_{0}^{\tau} \int_{\partial \Omega} u_{t t}^{N}(x, t)\left(\int_{0}^{t} \int_{\Omega} u_{\eta}^{N}(\xi, \eta) d \xi d \eta\right) d s_{x} d t \\
& \quad-\int_{0}^{\tau} \int_{\partial \Omega} u_{t t}^{N}(x, t)\left(\int_{0}^{t} \int_{\Omega} w^{N}(\xi, \eta) d \xi d \eta\right) d s_{x} d t
\end{aligned}
$$

With the same reasoning in (22), we find

$$
a\left(u_{t t t}^{N}, u_{t t}^{N}\right)_{L^{2}\left(Q_{\tau}\right)}=\frac{a}{2}\left\|u_{\tau \tau}^{N}(x, \tau)\right\|_{L^{2}(\Omega)}^{2}-\frac{a}{2}\left\|u_{t t}^{N}(x, 0)\right\|_{L^{2}(\Omega)}^{2},
$$




$$
\begin{aligned}
& \beta\left(u_{t t}^{N}, u_{t t}^{N}\right)_{L^{2}\left(Q_{\tau}\right)}=\beta \int_{0}^{\tau}\left\|u_{t t}^{N}(x, t)\right\|_{L^{2}(\Omega)}^{2} d t, \\
& c^{2}\left(\nabla u^{N}, \nabla u_{t t}^{N}\right)_{L^{2}\left(Q_{\tau}\right)}=c^{2}\left(\nabla u^{N}(x, \tau), \nabla u_{\tau}^{N}(x, \tau)\right)_{L^{2}\left(Q_{\tau}\right)} \\
& -c^{2}\left(\nabla u^{N}(x, 0), \nabla u_{\tau}^{N}(x, 0)\right)_{L^{2}(\Omega)} \\
& -c^{2} \int_{0}^{\tau}\left\|\nabla u_{t}^{N}(x, t)\right\|_{L^{2}(\Omega)}^{2} d t \\
& b\left(\nabla u_{t}^{N}, \nabla u_{t t}^{N}\right)_{L^{2}\left(Q_{\tau}\right)}=\frac{b}{2}\left\|\nabla u_{\tau}^{N}(x, \tau)\right\|_{L^{2}(\Omega)}^{2} \\
& -\frac{b}{2}\left\|\nabla u_{t}^{N}(x, 0)\right\|_{L^{2}(\Omega)}^{2} \\
& =\frac{1}{2}\left\{h^{\prime N}(\tau)\left\|\nabla u^{N}(x, \tau)\right\|_{L^{2}(\Omega)}^{2}-2\left(\nabla w^{N}(\tau), \nabla u_{\tau}^{N}\right)_{L^{2}(\Omega)}\right\} \\
& +\frac{1}{2} \int_{0}^{\tau} h^{\prime \prime N}(t) d t-\frac{1}{2} \int_{0}^{\tau} h^{\prime}(t)\left\|\nabla u^{N}(x, t)\right\|_{L^{2}(\Omega)}^{2} d t, \\
& c^{2} \int_{0}^{\tau} \int_{\partial \Omega} u_{t t}^{N}\left(\int_{0}^{\tau} \int_{\Omega} u^{N}(\xi, \eta) d \xi d \eta\right) d s_{x} d t \\
& =c^{2} \int_{\partial \Omega} u_{\tau}^{N}(x, \tau) \int_{0}^{\tau} \int_{\Omega} u^{N}(\xi, t) d \xi t d s_{x} \\
& -c^{2} \int_{\partial \Omega} \int_{0}^{\tau} u_{t}^{N}(x, t) \int_{\Omega} u^{N}(\xi, t) d \xi d t d s_{x} \\
& b \int_{0}^{\tau} \int_{\partial \Omega} u_{t t}^{N}(x, t)\left(\int_{0}^{t} \int_{\Omega} u_{\eta}^{N}(\xi, \eta) d \xi d \eta\right) d s_{x} d t \\
& =b \int_{\partial \Omega} u_{\tau}^{N}(x, \tau) \int_{\Omega} u^{N}(\xi, \tau) d \xi d s_{x} \\
& -b \int_{\partial \Omega} u_{\tau}^{N}(x, \tau) \int_{\Omega} u^{N}(\xi, 0) d \xi d s_{x} \\
& -b \int_{\partial \Omega} \int_{0}^{\tau} u_{t}^{N}(x, t) \int_{\Omega} u_{t}^{N}(\xi, t) d \xi d t d s . \\
& \int_{0}^{\tau} \int_{\partial \Omega} u_{t t}^{N}\left(\int_{0}^{t} \int_{\Omega} w^{N}(\xi, \eta) d \xi d \eta\right) d s_{x} d t \\
& =\int_{\partial \Omega} u_{\tau}^{N}(x, \tau) \int_{0}^{\tau} \int_{\Omega} w^{N}(\xi, t) d \xi d t d s_{x} \\
& -\int_{\partial \Omega} \int_{0}^{\tau} u_{t}^{N}(x, t) \int_{\Omega} w^{N}(\xi, t) d \xi d t d s_{x} .
\end{aligned}
$$

A substitution of equalities (33)-(40) in (22) gives

$$
\begin{aligned}
& \frac{a}{2}\left\|u_{\tau \tau}^{N}(x, \tau)\right\|_{L^{2}(\Omega)}^{2}+\frac{b}{2}\left\|\nabla u_{\tau}^{N}(x, \tau)\right\|_{L^{2}(\Omega)}^{2} \\
& +c^{2}\left(\nabla u^{N}(x, \tau), \nabla u_{\tau}^{N}(x, \tau)\right)_{L^{2}(\Omega)} \\
& -\frac{1}{2}\left\{h^{\prime N}(\tau)+h(\tau)\left\|\nabla u^{N}(x, \tau)\right\|_{L^{2}(\Omega)}^{2}\right. \\
& \left.-2\left(\nabla w^{N}(\tau), \nabla u_{\tau}^{N}\right)_{L^{2}(\Omega)}\right\} \\
& =\frac{a}{2}\left\|u_{t t}^{N}(x, 0)\right\|_{L^{2}(\Omega)}^{2}+c^{2}\left(\nabla u^{N}(x, 0), \nabla u_{t}^{N}(x, 0)\right)_{L^{2}(\Omega)} \\
& +\frac{b}{2}\left\|\nabla u_{t}^{N}(x, 0)\right\|_{L^{2}(\Omega)}^{2}-\beta \int_{0}^{\tau}\left\|u_{t t}^{N}(x, t)\right\|_{L^{2}(\Omega)}^{2} d t \\
& +c^{2} \int_{0}^{\tau}\left\|\nabla u_{t}(x, t)\right\|_{L^{2}(\Omega)}^{2}-\frac{1}{2} \int_{0}^{\tau} h^{\prime \prime N}(t) d t \\
& +\frac{1}{2} \int_{0}^{\tau} h^{\prime}(t)\left\|\nabla u^{N}(x, t)\right\|_{L^{2}(\Omega)}^{2} d t \\
& +c^{2} \int_{\partial \Omega} u_{\tau}^{N}(x, \tau) \int_{0}^{\tau} \int_{\Omega} u^{N}(\xi, t) d \xi d t d s_{x} \\
& -c^{2} \int_{\partial \Omega} \int_{0}^{\tau} u_{t}^{N}(x, t) \int_{\Omega} u^{N}(\xi, t) d \xi d t d s_{x} \\
& +b \int_{\partial \Omega} u_{\tau}^{N}(x, \tau) \int_{\Omega} u^{N}(\xi, \tau) d \xi d s_{x} \\
& -b \int_{\partial \Omega} u_{\tau}^{N}(x, \tau) \int_{\Omega} u^{N}(\xi, 0) d \xi d s_{x} \\
& -b \int_{\partial \Omega} \int_{0}^{\tau} u_{t}^{N}(x, t) \int_{\Omega} u_{t}^{N}(\xi, t) d \xi d t d s_{x} \\
& \cdot \int_{\partial \Omega} u_{\tau}^{N}(x, t) \int_{0}^{\tau} \int_{\Omega} w^{N}(\xi, t) d \xi d t d s_{x} \\
& -\int_{\partial \Omega} \int_{0}^{\tau} u_{t}^{N}(x, t) \int_{\Omega} w^{N}(\xi, t) d \xi d t d s_{x}+\left(F, u_{t t}^{N}\right)_{L^{2}\left(Q_{\tau}\right)} \text {. }
\end{aligned}
$$

Multiplying (32) by $\lambda$ and using (41), we get

$$
\begin{aligned}
\lambda a\left(u_{\tau \tau}^{N}, u_{\tau}^{N}\right)_{L^{2}(\Omega)}+\frac{\lambda \beta}{2}\left\|u_{\tau}^{N}(x, \tau)\right\|_{L^{2}(\Omega)}^{2} \\
+\frac{\lambda c^{2}}{2}\left\|\nabla u^{N}(x, \tau)\right\|_{L^{2}(\Omega)}^{2} \frac{\lambda}{2} h \circ \nabla u^{N}(\tau) \\
\quad-\frac{\lambda}{2} G(\tau)\left\|\nabla u^{N}(x, \tau)\right\|_{L^{2}(\Omega)}^{2} \frac{a}{2}\left\|u_{\tau \tau}^{N}(x, \tau)\right\|_{L^{2}(\Omega)}^{2} \\
+\frac{b}{2}\left\|\nabla u_{\tau}^{N}(x, \tau)\right\|_{L^{2}(\Omega)}^{2}+c^{2}\left(\nabla u^{N}(x, \tau), \nabla u_{\tau}^{N}(x, \tau)\right)_{L^{2}(\Omega)} \\
\quad-\frac{1}{2}\left\{h^{\prime N}(\tau)+h(\tau)\left\|\nabla u^{N}(x, \tau)\right\|_{L^{2}(\Omega)}^{2}\right. \\
\left.\quad-2\left(\nabla w^{N}(\tau), \nabla u_{\tau}^{N}\right)_{L^{2}(\Omega)}\right\} \\
=\lambda\left(F, u_{t}^{N}\right)_{L^{2}\left(Q_{\tau}\right)}+\left(F, u_{t t}^{N}\right)_{L^{2}\left(Q_{\tau}\right)} \\
+\lambda a\left(u_{t t}^{N}(x, 0), u_{t}^{N}(x, 0)\right)_{L^{2}(\Omega)} \\
+(\lambda a-\beta) \int_{0}^{\tau}\left\|u_{t t}(x, t)\right\|_{L^{2}(\Omega)}^{2} d t
\end{aligned}
$$




$$
\begin{aligned}
& +\left(c^{2}-\lambda b\right) \int_{0}^{\tau}\left\|\nabla u_{t}^{N}(x, t)\right\|_{L^{2}(\Omega)}^{2} d t+\frac{\lambda}{2} \int_{0}^{\tau} h^{\prime N}(t) d t \\
& -\frac{\lambda}{2} \int_{0}^{\tau} h(t)\left\|\nabla u^{N}(x, t)\right\|_{L^{2}(\Omega)}^{2} d t \\
& +\lambda c^{2} \int_{\partial \Omega} u^{N}(x, \tau) \int_{0}^{\tau} \int_{\Omega} u^{N}(\xi, t) d \xi d t d s_{x} \\
& +\frac{\lambda c^{2}}{2}\left\|\nabla u^{N}(x, 0)\right\|_{L^{2}(\Omega)}^{2} \\
& -\lambda c^{2} \int_{\partial \Omega} \int_{0}^{\tau} u^{N}(x, t) \int_{\Omega} u^{N}(\xi, t) d \xi d t d s_{x} \\
& +\frac{\lambda \beta}{2}\left\|u_{t}^{N}(x, 0)\right\|_{L^{2}(\Omega)}^{2} \\
& +\left(\lambda b-c^{2}\right) \int_{\partial \Omega} \int_{0}^{\tau} u_{t}^{N}(x, t) \int_{\Omega} u^{N}(\xi, t) d \xi d t d s_{x} \\
& -\lambda b \int_{\partial \Omega} \int_{0}^{\tau} u_{t}^{N}(x, t) \int_{\Omega} u^{N}(\xi, 0) d \xi d t d s_{x} \\
& +\lambda \int_{\partial \Omega} u^{N}(x, \tau) \int_{0}^{\tau} \int_{\Omega} w^{N}(\xi, t) d \xi d t d s_{x} \\
& -\lambda \int_{\partial \Omega} \int_{0}^{\tau} u^{N}(x, t) \int_{\Omega} w^{N}(\xi, t) d \xi d t d s_{x} \\
& +\frac{a}{2}\left\|u_{t t}^{N}(x, 0)\right\|_{L^{2}(\Omega)}^{2}+c^{2}\left(\nabla u^{N}(x, 0), \nabla u_{t}^{N}(x, 0)\right)_{L^{2}(\Omega)} \\
& +\frac{b}{2}\left\|\nabla u_{t}^{N}(x, 0)\right\|_{L^{2}(\Omega)}^{2}-\frac{1}{2} \int_{0}^{\tau} h^{\prime \prime N}(t) d t \\
& +\frac{1}{2} \int_{0}^{\tau} h^{\prime}(t)\left\|\nabla u^{N}(x, t)\right\|_{L^{2}(\Omega)}^{2} d t \\
& +c^{2} \int_{\partial \Omega} u_{\tau}^{N}(x, \tau) \int_{0}^{\tau} \int_{\Omega} u^{N}(\xi, t) d \xi d t d s_{x} \\
& +b \int_{\partial \Omega} u_{\tau}^{N}(x, \tau) \int_{\Omega} u^{N}(x, \tau) d \xi d s_{x} \\
& -b \int_{\partial \Omega} u_{\tau}^{N}(x, \tau) \int_{\Omega} u^{N}(\xi, 0) d \xi d s_{x} \\
& -b \int_{\partial \Omega} \int_{0}^{\tau} u_{t}^{N}(\xi, t) d \xi d t d s_{x} \int_{\partial \Omega} \\
& \cdot u_{\tau}^{N}(x, \tau) \int_{0}^{\tau} \int_{\Omega} w^{N}(\xi, t) d \xi d t d s_{x} \\
& -\int_{\partial \Omega} \int_{0}^{\tau} u_{\tau}^{N}(x, t) \int_{\Omega} w^{N}(\xi, t) d \xi d t d s_{x} .
\end{aligned}
$$

where $0<\lambda<1$.

With the help of Cauchy and the trace inequalities, we can estimate all the terms in the RHS of (42) that gives

$$
\begin{aligned}
c^{2} \lambda & \int_{\partial \Omega} u^{N}(x, \tau) \int_{0}^{\tau} \int_{\Omega} u^{N}(\xi, t) d \xi d t d s_{x} \\
\leq & \frac{c^{2} \lambda}{2 \varepsilon_{1}}\left(\varepsilon\left\|\nabla u^{N}(x, \tau)\right\|_{L^{2}(\Omega)}^{2}+l(\varepsilon)\left\|u^{N}(x, \tau)\right\|_{L^{2}(\Omega)}^{2}\right) \\
& +\frac{c^{2} \lambda}{2} \varepsilon_{1} T|\Omega|\left\|\partial \Omega \mid \int_{0}^{\tau}\right\| u^{N}(x, t) \|_{L^{2}(\Omega)}^{2} d t,
\end{aligned}
$$

$$
\begin{aligned}
& -c^{2} \lambda \int_{\partial \Omega} \int_{0}^{\tau} u^{N}(x, t) \int_{\Omega} u^{N}(\xi, t) d \xi d t d s_{x} \\
& \leq \frac{c^{2} \lambda}{2} \varepsilon \int_{0}^{\tau}\left\|\nabla u^{N}(x, t)\right\|_{L^{2}(\Omega)}^{2} d t \\
& +\frac{c^{2} \lambda}{2}(l(\varepsilon)+|\Omega||\partial \Omega|) \int_{0}^{\tau}\left\|u^{N}(x, t)\right\|_{L^{2}(\Omega)}^{2} d t, \\
& \left(b \lambda-c^{2}\right) \int_{\partial \Omega} \int_{0}^{\tau} u_{t}^{N}(x, t) \int_{\Omega} u^{N}(\xi, \tau) d \xi d t d s_{x} \\
& \leq \frac{\left(b \lambda-c^{2}\right)}{2}\left(\varepsilon \int_{0}^{\tau}\left\|\nabla u_{t}^{N}(x, t)\right\|_{L^{2}(\Omega)}^{2} d t\right. \\
& \left.+l(\varepsilon) \int_{0}^{\tau}\left\|\nabla u_{t}^{N}(x, t)\right\|_{L^{2}(\Omega)}^{2} d t\right) \\
& +\frac{\left(b \lambda-c^{2}\right)}{2}|\Omega||\partial \Omega| \int_{0}^{\tau}\left\|u^{N}(x, t)\right\|_{L^{2}(\Omega)}^{2} d t, \\
& -b \lambda \int_{\partial \Omega} \int_{0}^{\tau} u_{t}^{N}(x, t) \int_{\Omega} u^{N}(\xi, 0) d \xi d t d s_{x} \\
& \leq \frac{b \lambda}{2}\left(\varepsilon \int_{0}^{\tau}\left\|\nabla u_{t}^{N}(x, t)\right\|_{L^{2}(\Omega)}^{2} d t+l(\varepsilon) \int_{0}^{\tau}\left\|u_{t}^{N}(x, t)\right\|_{L^{2}(\Omega)}^{2} d t\right) \\
& +\frac{b \lambda}{2}|\Omega||\partial \Omega| T\left\|u^{N}(x, 0)\right\|_{L^{2}(\Omega)}^{2}, \\
& c^{2} \int_{\partial \Omega} u_{\tau}^{N}(x, \tau) \int_{0}^{\tau} \int_{\Omega} u^{N}(\xi, t) d \xi d t d s_{x} \\
& \leq \frac{c^{2}}{2}\left(\frac{\varepsilon}{\varepsilon_{2}}\left\|\nabla u_{\tau}^{N}(x, \tau)\right\|_{L^{2}(\Omega)}^{2}+\frac{l(\varepsilon)}{\varepsilon_{2}}\left\|u_{\tau}^{N}(x, \tau)\right\|_{L^{2}(\Omega)}^{2}\right) \\
& +\frac{c^{2}}{2} \varepsilon_{2}|\Omega||\partial \Omega| T \int_{0}^{\tau}\left\|u^{N}(x, t)\right\|_{L^{2}(\Omega)}^{2} d t, \\
& +b \int_{\partial \Omega} u_{\tau}^{N}(x, \tau) \int_{\Omega} u^{N}(\xi, \tau) d \xi d s_{x} \\
& \leq \frac{b}{2 \varepsilon_{3}}\left(\varepsilon\left\|\nabla u_{\tau}^{N}(x, \tau)\right\|_{L^{2}(\Omega)}^{2}+l(\varepsilon)\left\|u_{\tau}^{N}(x, \tau)\right\|_{L^{2}(\Omega)}^{2}\right) \\
& +\frac{b}{2} \varepsilon_{3}|\Omega||\partial \Omega|\left\|u^{N}(x, \tau)\right\|_{L^{2}(\Omega)}^{2}, \\
& -b \int_{\partial \Omega} u_{\tau}^{N}(x, \tau) \int_{\Omega} u^{N}(\xi, 0) d \xi d s_{x} \\
& \leq \frac{b}{2 \varepsilon_{4}}\left(\varepsilon\left\|\nabla u_{\tau}^{N}(x, \tau)\right\|_{L^{2}(\Omega)}^{2}+l(\varepsilon)\left\|u_{\tau}^{N}(x, \tau)\right\|_{L^{2}(\Omega)}^{2}\right) \\
& +\frac{b}{2} \varepsilon_{4}|\Omega||\partial \Omega|\left\|u^{N}(x, \tau)\right\|_{L^{2}(\Omega)}^{2}, \\
& -b \int_{\partial \Omega} u_{\tau}^{N}(x, t) \int_{\Omega} u_{\tau}^{N}(\xi, \tau) d \xi d t d s_{x} \\
& \leq \frac{b}{2} \int_{0}^{\tau}\left\|\nabla u_{\tau}^{N}(x, \tau)\right\|_{L^{2}(\Omega)}^{2} d t \\
& +\frac{b}{2}(l(\varepsilon)|\Omega||\partial \Omega|) \int_{0}^{\tau}\left\|u_{\tau}^{N}(x, t)\right\|_{L^{2}(\Omega)}^{2} d t,
\end{aligned}
$$




$$
\begin{aligned}
& -\frac{\lambda a}{2}\left\|u_{\tau \tau}^{N}(x, \tau)\right\|_{L^{2}(\Omega)}^{2}-\frac{\lambda a}{2}\left\|u_{\tau \tau}^{N}(x, \tau)\right\|_{L^{2}(\Omega)}^{2} \\
& \leq \lambda a\left(u_{\tau \tau}^{N}(x, \tau), u_{\tau}^{N}(x, \tau)\right)_{L^{2}(\Omega)}, \\
& -\frac{c^{2} \varepsilon_{7}}{2}\left\|\nabla u^{N}(x, \tau)\right\|_{L^{2}(\Omega)}^{2}-\frac{c^{2}}{2 \varepsilon_{7}}\left\|\nabla u_{\tau}^{N}(x, \tau)\right\|_{L^{2}(\Omega)}^{2} \\
& \leq c^{2}\left(u^{N}(x, \tau), \nabla u_{\tau}^{N}(x, \tau)\right)_{L^{2}(\Omega)}, \\
& \lambda a\left(u_{t t}^{N}(x, 0), u_{\tau}^{N}(x, 0)\right) \\
& \leq \frac{\lambda a}{2}\left\|u_{t t}^{N}(x, 0)\right\|_{L^{2}(\Omega)}^{2}+\frac{\lambda a}{2}\left\|u_{\tau}^{N}(x, 0)\right\|_{L^{2}(\Omega)}^{2}, \\
& c^{2}\left(\nabla u^{N}(x, 0), u_{\tau}^{N}(x, 0)\right)_{L^{2}(\Omega)} \\
& \leq \frac{c^{2}}{2}\left\|\nabla u^{N}(x, 0)\right\|_{L^{2}(\Omega)}^{2}+\frac{c^{2}}{2}\left\|\nabla u_{\tau}^{N}(x, 0)\right\|_{L^{2}(\Omega)}^{2} . \\
& -\frac{G(\tau)}{2}\left(\frac{1}{\varepsilon_{8}}+\frac{1}{\varepsilon_{9}}\right)\left\|\nabla u^{N}(x, \tau)\right\|_{L^{2}(\Omega)}^{2}-\frac{\varepsilon_{8}}{2} h \circ \nabla u^{N}(\tau) \\
& -\frac{\varepsilon_{9}}{2} G(\tau)\left\|\nabla u^{N}(x, \tau)\right\|_{L^{2}(\Omega)}^{2} \\
& \leq 2\left(\nabla w^{N}(x, \tau), \nabla u_{\tau}^{N}(x, \tau)\right)_{L^{2}(\Omega)}, \\
& \int_{\partial \Omega} u_{\tau}^{N}(x, \tau) \int_{0}^{\tau} \int_{\Omega} w^{N}(\xi, t) d \xi d t d s_{x} \\
& \leq\left(\frac{1}{2 \varepsilon_{5}}+\frac{1}{2 \varepsilon_{5}^{\prime}}\right)\left(\varepsilon\left\|\nabla u_{\tau}^{N}(x, \tau)\right\|_{L^{2}(\Omega)}^{2}+l(\varepsilon)\left\|u_{\tau}^{N}(x, \tau)\right\|_{L^{2}(\Omega)}^{2}\right) \\
& +|\Omega||\partial \Omega| T\left(\frac{\varepsilon_{5}}{2} \int_{0}^{\tau} h \circ u^{N}(t) d t+\frac{\varepsilon_{5}^{\prime}}{2} h_{0} \int_{0}^{\tau}\left\|u^{N}(x, t)\right\|_{L^{2}(\Omega)}^{2}\right),
\end{aligned}
$$

$$
\begin{aligned}
& \lambda \int_{\partial \Omega} u^{N}(x, \tau) \int_{0}^{\tau} \int_{\Omega} w^{N}(\xi, t) d \xi d t d s_{x} \\
& \leq \lambda\left(\frac{1}{2 \varepsilon_{6}}+\frac{1}{2 \varepsilon_{6}^{\prime}}\right)\left(\varepsilon\left\|\nabla u^{N}(x, \tau)\right\|_{L^{2}(\Omega)}^{2}+l(\varepsilon)\left\|u^{N}(x, \tau)\right\|_{L^{2}(\Omega)}^{2}\right) \\
& \quad+\lambda|\Omega \| \partial \Omega| T\left(\frac{\varepsilon_{6}}{2} \int_{0}^{\tau} h \circ u^{N}(t) d t+\frac{\varepsilon_{6}^{\prime}}{2} h_{0} \int_{0}^{\tau}\left\|u^{N}(x, \tau)\right\|_{L^{2}(\Omega)}^{2}\right),
\end{aligned}
$$

$$
\begin{aligned}
& \int_{\partial \Omega} \int_{0}^{\tau} u_{t}^{N}(x, \tau) \int_{\Omega} w^{N}(\xi, t) d \xi d t d s_{x} \\
& \leq \quad\left(\varepsilon \int_{0}^{\tau}\left\|\nabla u_{t}^{N}(x, t)\right\|_{L^{2}(\Omega)}^{2} d t+l(\varepsilon) \int_{0}^{\tau}\left\|\nabla u_{t}^{N}(x, t)\right\|_{L^{2}(\Omega)}^{2} d t\right) \\
& \quad+\frac{1}{2}|\Omega||\partial \Omega| T\left(\int_{0}^{\tau} h \circ u^{N}(t) d t+h_{0} \int_{0}^{\tau}\left\|u^{N}(x, t)\right\|_{L^{2}(\Omega)}^{2} d t\right)
\end{aligned}
$$

$$
\begin{aligned}
\lambda \int_{\partial \Omega} \int_{0}^{\tau} u^{N}(x, t) \int_{\Omega} u^{N}(\xi, t) d \xi d t d s_{x} \\
\leq \lambda\left(\varepsilon \int_{0}^{\tau}\left\|\nabla u^{N}(x, t)\right\|_{L^{2}(\Omega)}^{2} d t+l(\varepsilon) \int_{0}^{\tau}\left\|u^{N}(x, t)\right\|_{L^{2}(\Omega)}^{2} d t\right) \\
\quad+\frac{\lambda}{2}|\Omega \| \partial \Omega| T\left(\int_{0}^{\tau} h \circ u^{N}(t) d t+h_{0} \int_{0}^{\tau}\left\|u^{N}(x, t)\right\|_{L^{2}(\Omega)}^{2} d t\right),
\end{aligned}
$$

$$
\lambda\left(F, u_{\tau}^{N}\right)_{L^{2}\left(Q_{\tau}\right)} \leq \frac{\lambda}{2} \int_{0}^{\tau}\|F(x, t)\|_{L^{2}(\Omega)}^{2} d t+\frac{1}{2} \int_{0}^{\tau}\left\|u_{t t}(x, t)\right\|_{L^{2}(\Omega)}^{2} d t .
$$

Combining inequalities (45)-(60) and equality (44) and make use of the following inequality

$$
\begin{aligned}
& m_{1}\left\|u^{N}(x, \tau)\right\|_{L^{2}(\Omega)}^{2} \\
& \quad \leq m_{1}\left\|u^{N}(x, t)\right\|_{L^{2}(\Omega)}^{2}+m_{1}\left\|u_{\tau}^{N}(x, t)\right\|_{L^{2}(\Omega)}^{2} \\
& \quad+m_{1}\left\|u^{N}(x, 0)\right\|_{L^{2}(\Omega)}^{2} \\
& m_{2}\left\|u_{t}^{N}(x, \tau)\right\|_{L^{2}(\Omega)}^{2} \\
& \quad \leq m_{2}\left\|u_{t}^{N}(x, t)\right\|_{L^{2}(\Omega)}^{2}+m_{2}\left\|u_{t t}^{N}(x, t)\right\|_{L^{2}(\Omega)}^{2} \\
& \quad+m_{2}\left\|u_{t}^{N}(x, 0)\right\|_{L^{2}(\Omega)}^{2} \\
& m_{3}\left\|\nabla u^{N}(x, \tau)\right\|_{L^{2}(\Omega)}^{2} \\
& \leq m_{3}\left\|\nabla u^{N}(x, t)\right\|_{L^{2}\left(Q_{\tau}\right)}^{2}+m_{3}\left\|\nabla u_{t}^{N}(x, t)\right\|_{L^{2}\left(Q_{\tau}\right)}^{2} \\
& \quad+m_{3}\left\|\nabla u^{N}(x, 0)\right\|_{L^{2}(\Omega)}^{2} \\
& h \circ u^{N}(\tau) \\
& \leq \frac{1}{2} h \circ u^{N}(t) d t+\frac{h_{0}}{2}\left\|u_{t}^{N}(x, t)\right\|_{L^{2}\left(Q_{\tau}\right)}^{2}-\int_{0}^{\tau} h^{\prime N}(t) d t, \\
& h \circ \nabla u^{N}(\tau) \\
& \leq \frac{1}{2} \int_{0}^{\tau} h \circ \nabla u^{N}(t) d t+\frac{h_{0}}{2}\left\|\nabla u_{t}^{N}(x, t)\right\|_{L^{2}\left(Q_{\tau}\right)}^{2}-\int_{0}^{\tau} h^{\prime N}(t) d t
\end{aligned}
$$

where

$$
\begin{aligned}
& m_{1}:=\frac{c^{2} \lambda}{\varepsilon_{1}} l(\varepsilon)+\frac{b}{2} \varepsilon_{3}|\Omega||\partial \Omega|+\lambda\left(\frac{1}{2 \varepsilon_{6}}+\frac{1}{2 \varepsilon_{6}^{\prime}}\right) l(\varepsilon), \\
& m_{2}:=l(\varepsilon)\left(\frac{c^{2}}{2 \varepsilon_{2}}+\frac{b}{2 \varepsilon_{2}}+\frac{b}{2 \varepsilon_{4}}+\left(\frac{1}{2 \varepsilon_{5}}+\frac{1}{2 \varepsilon_{5}^{\prime}}\right)\right), \\
& m_{3}:=\frac{c^{2} \varepsilon_{7}}{2}+h(0)+\frac{c^{2} \lambda}{2 \varepsilon_{1}}+\frac{c^{2} \varepsilon_{9}}{2}+\lambda\left(\frac{1}{2 \varepsilon_{6}}+\frac{1}{2 \varepsilon_{6}^{\prime}}\right) \varepsilon,
\end{aligned}
$$


and we have

$$
\begin{aligned}
& \frac{c^{2} \lambda}{2 \varepsilon_{1}} l(\varepsilon)\left\|u^{N}(x, \tau)\right\|_{L^{2}(\Omega)}^{2}+\frac{\lambda}{2}(\beta-a)\left\|u_{t}^{N}(x, \tau)\right\|_{L^{2}(\Omega)}^{2} \\
& +\frac{\lambda}{2}\left(c^{2}-G(\tau)\right)\left\|\nabla u^{N}(x, \tau)\right\|_{L^{2}(\Omega)}^{2} \\
& +\left\{\frac{b}{2}-\frac{c^{2} \varepsilon}{2 \varepsilon_{2}}-\frac{b \varepsilon}{2 \varepsilon_{3}}-\frac{b \varepsilon}{2 \varepsilon_{4}}-\frac{c^{2}}{2 \varepsilon_{7}}-\frac{\varepsilon}{2 \varepsilon_{5}}\right. \\
& \left.-\frac{\varepsilon}{2 \varepsilon_{5}^{\prime}}-\frac{G(\tau)}{2}\left(\frac{1}{\varepsilon_{8}}+\frac{1}{\varepsilon_{9}}\right)\right\}\left\|\nabla u_{\tau}^{N}(x, \tau)\right\|_{L^{2}(\Omega)}^{2} \\
& +\left(\frac{a}{2}-\frac{\lambda a}{2}\right)\left\|u_{\tau \tau}^{N}(x, \tau)\right\|_{L^{2}(\Omega)}^{2} \\
& +\left(\frac{\lambda+1}{2}\right) h \circ \nabla u^{N}(\tau)+h \circ u^{N}(\tau) \\
& \leq \gamma_{1} \int_{0}^{\tau}\left\|u^{N}(x, t)\right\|_{L^{2}(\Omega)}^{2} d t+\gamma_{2} \int_{0}^{\tau}\left\|u_{t}^{N}(x, t)\right\|_{L^{2}(\Omega)}^{2} d t \\
& +\underbrace{\left\{\frac{c^{2} \lambda}{2} \varepsilon+\lambda \varepsilon+m_{3}\right\}}_{\alpha_{1}} \int_{0}^{\tau}\left\|\nabla u^{N}(x, t)\right\|_{L^{2}(\Omega)}^{2} d t \\
& +\gamma_{3} \int_{0}^{\tau}\left\|\nabla u_{\tau}^{N}(x, t)\right\|_{L^{2}(\Omega)}^{2} d t+\underbrace{\frac{(\lambda+3) \xi}{2}}_{\alpha_{2}} \int_{0}^{\tau} h \circ \nabla u^{N}(\tau) \\
& +\gamma_{4} \int_{0}^{\tau} h \circ u^{N}(\tau)+\underbrace{\left\{\frac{\lambda a+\beta+m_{2}}{2}\right\}}_{\alpha_{3}} \int_{0}^{\tau}\left\|u_{t t}^{N}(x, t)\right\|_{L^{2}(\Omega)}^{2} d t \\
& +\underbrace{\left\{\frac{b \lambda}{2}|\Omega||\partial \Omega| T+\frac{b}{2} \varepsilon_{4}|\Omega||\partial \Omega|+m_{1}\right\}}_{\alpha_{4}}\left\|u^{N}(x, 0)\right\|_{L^{2}(\Omega)}^{2} \\
& +\underbrace{\left\{\frac{\lambda a}{2}+\frac{\lambda \beta}{2}+m_{2}\right\}}_{\alpha_{5}}\left\|u_{t}^{N}(x, 0)\right\|_{L^{2}(\Omega)}^{2} \\
& +\left(\frac{\lambda+1}{2}\right) \int_{0}^{\tau}\|F(x, t)\|_{L^{2}(\Omega)}^{2} d t \\
& +\underbrace{\left\{\frac{\lambda}{2} c^{2}+\frac{c^{2}}{2}+\frac{1}{2} h(0)+m_{3}\right\}}_{\alpha_{6}}\left\|\nabla u^{N}(x, 0)\right\|_{L^{2}(\Omega)}^{2} \\
& +\underbrace{\left\{\frac{b}{2}+\frac{c^{2}}{2}\right\}}_{\alpha_{7}}\left\|\nabla u_{t}^{N}(x, 0)\right\|_{L^{2}(\Omega)}^{2} \\
& +\underbrace{\left\{\frac{\lambda a}{2}+\frac{a}{2}\right\}}_{\alpha_{7}}\left\|\nabla u_{t t}^{N}(x, 0)\right\|_{L^{2}(\Omega)}^{2},
\end{aligned}
$$

where

$$
\begin{aligned}
\gamma_{1}:= & \left\{\frac{c^{2} \lambda}{2} \varepsilon_{1} T|\partial \Omega||\Omega|+\frac{c^{2} \lambda}{2}(l(\varepsilon)+|\partial \Omega||\Omega|)\right. \\
& +\frac{\left(b \lambda+c^{2}\right)}{2}|\partial \Omega||\Omega|+\left(\frac{c^{2}}{2} \varepsilon_{2}+\frac{c^{2}}{2} \varepsilon_{5}^{\prime} h_{0}+\frac{\lambda+1}{2}\right) \\
& \left.\cdot|\partial \Omega||\Omega| T+\lambda l(\varepsilon)+m_{1}\right\},
\end{aligned}
$$

$$
\begin{aligned}
\gamma_{2}:= & \frac{\left(b \lambda+c^{2}\right)}{2} l(\varepsilon)+\frac{b \lambda}{2} l(\varepsilon)+\frac{b}{2}(l(\varepsilon)+|\partial \Omega||\Omega|)+l(\varepsilon) \\
+ & \left.\frac{h_{0}}{2 \xi}+\frac{\lambda}{2}+m_{2}\right\} \\
\gamma_{3}:= & \left\{c^{2}+\lambda b+\frac{\left(b \lambda+c^{2}\right)}{2} \varepsilon+\frac{b(\lambda+1)}{2} \varepsilon\right. \\
& \left.+\lambda \varepsilon+\frac{h_{0} \lambda}{2}+\frac{h_{0}}{2 \xi}+m_{3}\right\}, \\
\gamma_{4}:= & \left\{\left(\frac{\varepsilon_{5}}{2}+\frac{\lambda+1}{2}\right)|\partial \Omega||\Omega| T+\frac{3 \xi}{2}\right\}
\end{aligned}
$$

Choosing $\varepsilon_{2}, \varepsilon_{3}, \varepsilon_{4}, \varepsilon_{5}, \varepsilon_{5}^{\prime}, \varepsilon_{7}, \varepsilon_{8}$ and $\varepsilon_{9}$ sufficiently large

$$
\begin{aligned}
\alpha_{0}= & \frac{b}{2}-\frac{c^{2} \varepsilon}{2 \varepsilon_{2}}-\frac{b \varepsilon}{2 \varepsilon_{3}}-\frac{b \varepsilon}{2 \varepsilon_{4}}-\frac{c^{2}}{2 \varepsilon_{7}}-\frac{\varepsilon}{2 \varepsilon_{5}} \\
& -\frac{\varepsilon}{2 \varepsilon_{5}^{\prime}}-\frac{G(\tau)}{2}\left(\frac{1}{\varepsilon_{8}}+\frac{1}{\varepsilon_{9}}\right)>0 .
\end{aligned}
$$

By using (2)-(4), the relation (64) reduces to

$$
\begin{aligned}
\left\|u^{N}(x, \tau)\right\|_{L^{2}(\Omega)}^{2}+\left\|\nabla u^{N}(x, \tau)\right\|_{L^{2}(\Omega)}^{2} & +\left\|u_{\tau}^{N}(x, \tau)\right\|_{L^{2}(\Omega)}^{2}+\left\|\nabla u_{\tau}^{N}(x, \tau)\right\|_{L^{2}(\Omega)}^{2} \\
& +\left\|u_{\tau \tau}^{N}(x, \tau)\right\|_{L^{2}(\Omega)}^{2}+h \circ u^{N}(\tau)+h \circ \nabla u^{N}(\tau) \\
\leq & D \int_{0}^{\tau}\left\{\left\|u^{N}(x, t)\right\|_{L^{2}(\Omega)}^{2}+\left\|\nabla u^{N}(x, t)\right\|_{L^{2}(\Omega)}^{2}\right. \\
& +\left\|u_{t}^{N}(x, t)\right\|_{L^{2}(\Omega)}^{2}+\left\|\nabla u_{t}^{N}(x, t)\right\|_{L^{2}(\Omega)}^{2}+\left\|u_{t t}^{N}(x, t)\right\|_{L^{2}(\Omega)}^{2} \\
& \left.+h \circ u^{N}(t)+h \circ \nabla u^{N}(t)+\|F\|_{L^{2}(\Omega)}^{2}\right\} d t \\
& +D\left(\left\|u^{N}(x, 0)\right\|_{W_{2}^{1}(\Omega)}^{2}+\left\|u_{t}^{N}(x, 0)\right\|_{W_{2}^{1}(\Omega)}^{2}\right. \\
& \left.+\left\|u_{t t}^{N}(x, 0)\right\|_{L^{2}(\Omega)}^{2}\right), \\
\leq & D \int_{0}^{\tau}\left\{\left\|u^{N}(x, t)\right\|_{L^{2}(\Omega)}^{2}+\left\|u_{t t}^{N}(x, t)\right\|_{L^{2}(\Omega)}^{2}\right. \\
& \left.+h \circ u^{N}(t)+h \circ \nabla u^{N}(t)+\|F\|_{L^{2}(\Omega)}^{2}\right\} d t \\
& +D\left(\left\|u^{N}(x, 0)\right\|_{W_{2}^{1}(\Omega)}^{2}+\left\|u_{t}^{N}(x, 0)\right\|_{W_{2}^{1}(\Omega)}^{2}\right. \\
& \left.+\left\|u_{t t}^{N}(x, 0)\right\|_{L^{2}(\Omega)}^{2}+h \circ u^{N}(0)+h \circ \nabla u^{N}(0)\right)
\end{aligned}
$$


where

$$
D:=\frac{\max \left\{\alpha_{i}, i=1 \cdots 8\right\}}{\min \left\{\left(c^{2} \lambda / 2 \varepsilon_{1}\right) l(\varepsilon),(\lambda / 2)(\beta-a),(\lambda / 2)\left(c^{2}-G(\tau)\right),(a / 2)(1-\lambda),(\lambda+1 / 2), 1, \alpha_{0}\right\}} .
$$

Using the inequality of Gronwall to (67) and integrating the result from 0 to $\tau$ that gives

$$
\begin{aligned}
\left\|u^{N}(x, t)\right\|_{W_{2}^{1}\left(Q_{\tau}\right)}^{2}+\left\|u_{t}^{N}(x, t)\right\|_{W_{2}^{1}\left(Q_{\tau}\right)}^{2} & +\left\|u^{N}(x, t)\right\|_{h, W_{2}^{1}\left(Q_{\tau}\right)}^{2}+\left\|u_{t t}^{N}(x, t)\right\|_{L^{2}\left(Q_{\tau}\right)}^{2} \\
\leq & D e^{D T}\left\{\left\|u_{0}(x)\right\|_{W_{2}^{1}(\Omega)}^{2}+\left\|u_{1}(x)\right\|_{W_{2}^{1}(\Omega)}^{2}\right. \\
& \left.+\left\|u_{2}(x)\right\|_{L^{2}(\Omega)}^{2}+\|F\|_{L^{2}(\Omega)}^{2}\right\} .
\end{aligned}
$$

where

$$
\begin{aligned}
& \|u(x, t)\|_{h, W_{2}^{1}\left(Q_{\tau}\right)}^{2}:=\int_{0}^{\tau} h \circ u(t) d t+\int_{0}^{\tau} h \circ \nabla u(t) d t, \\
& \|u(x, 0)\|_{h, W_{2}^{1}\left(Q_{\tau}\right)}^{2}=0 .
\end{aligned}
$$

We deduce from (69) that

$$
\begin{aligned}
& \left\|u^{N}(x, t)\right\|_{w_{2}^{1}\left(Q_{\tau}\right)}^{2}+\left\|u_{t}^{N}(x, t)\right\|_{w_{2}^{1}\left(Q_{\tau}\right)}^{2} \\
& \quad+\left\|u^{N}(x, t)\right\|_{h, W_{2}^{1}\left(Q_{\tau}\right)}^{2}+\left\|u_{t t}^{N}(x, t)\right\|_{L^{2}\left(Q_{\tau}\right)}^{2} \leq A .
\end{aligned}
$$

Hence, $\left\{u^{N}\right\}_{N \geq 1}$ is sequence bounded in $V\left(Q_{T}\right)$, and we can extract from it a subsequence for which we use the same notation which converges weakly in $V\left(Q_{T}\right)$ to a limit function $u(x, t)$, and we have to show that $u(x, t)$ is a generalized solution of (1). Since $u^{N}(x, t) \longrightarrow u(x, t)$ in $L^{2}\left(Q_{T}\right)$ and $u^{N}(x, 0) \longrightarrow \zeta(x)$ in $L^{2}(\Omega)$, then $u(x, 0)=\zeta(x)$.

Now to prove that (15) holds, we multiply each of the relations (15) by a function $p_{l}(t) \in W_{2}^{1}(0, T), p_{l}(T)=0$. Hence, collect them the obtained equalities ranging from $l=1$ to $l=N$ and integrating the result over $t$ on $(0, T)$. If we let $\eta^{N}=\sum_{k=1}^{N} p_{l}(t) Z_{k}(x)$, then we have

$$
\begin{aligned}
a\left(u_{t t}^{N}, \eta_{t}^{N}\right)_{L^{2}\left(Q_{T}\right)}+\beta\left(u_{t}^{N}, \eta_{t}^{N}\right)_{L^{2}\left(Q_{T}\right)}-c^{2}\left(\nabla u^{N}, \nabla \eta^{N}\right)_{L^{2}\left(Q_{T}\right)} & -b\left(\nabla u_{t}^{N}, \nabla \eta^{N}\right)_{L^{2}\left(Q_{T}\right)}-\left(\nabla w^{N}, \nabla \eta^{N}\right)_{L^{2}\left(Q_{T}\right)} \\
= & a\left(u_{t t}^{N}(x, 0), \eta^{N}(0)\right)_{L^{2}(\Omega)}+\beta\left(u_{t}^{N}(x, 0), \eta^{N}(0)\right)_{L^{2}(\Omega)} \\
& +c^{2} \int_{\partial \Omega} \int_{0}^{T} \eta^{N}(x, t)\left(\int_{0}^{t} \int_{\Omega} u^{N}(\xi, \tau) d \xi d \tau\right) d t d s_{x} \\
& +b \int_{\partial \Omega} \int_{0}^{T} \eta^{N}(x, t) \int_{\Omega} u^{N}(\xi, \tau) d \xi d t d s_{x} \\
& -b \int_{\partial \Omega} \int_{0}^{T} \eta^{N}(x, t) \int_{\Omega} u^{N}(\xi, 0) d \xi d t d s_{x}
\end{aligned}
$$

$$
\begin{aligned}
& +\int_{\partial \Omega} \int_{0}^{T} \eta^{N}(x, t)\left(\int_{0}^{t} \int_{\Omega} w^{N}(\xi, \tau) d \xi d \tau\right) d t d s_{x} \\
& +\left(F, \eta_{t}^{N}\right)_{L^{2}\left(Q_{T}\right)}
\end{aligned}
$$

for all $\eta^{N}$ of the form $\sum_{k=1}^{N} p_{l}(t) Z_{k}(x)$ and $\alpha>0$.

Since

$$
\begin{gathered}
\int_{0}^{t} \int_{\Omega}\left(\left(u^{N}(\xi, \tau)-u(\xi, \tau)\right) d \xi d \tau\right) \leq \sqrt{T|\Omega|}\left\|u^{N}-u\right\|_{L^{2}\left(Q_{T}\right)}, \\
\left\|u^{N}-u\right\|_{L^{2}\left(Q_{T}\right)} \rightarrow 0 \text { as } N \rightarrow \infty .
\end{gathered}
$$

Thus, the limit function $u$ satisfies (15) for every $\eta^{N}=$ $\sum_{k=1}^{N} p_{l}(t) Z_{k}(x)$.

We define the totality of all functions of the form $\eta^{N}=$ $\sum_{k=1}^{N} p_{l}(t) Z_{k}(x)$ by $\mathbb{Q}_{N}$, with $p_{l}(t) \in W_{2}^{1}(0, T), p_{l}(T)=0$.

But $\cup_{l=1}^{N} \mathbb{Q}_{N}$ is dense in $W\left(Q_{T}\right)$, hence the relation (15) holds $\forall u \in W\left(Q_{T}\right)$. Then, we have shown that the limit function $u(x, t)$ is a generalized solution of problem (1) in $V\left(Q_{T}\right)$.

\section{Uniqueness of the Problem}

Theorem 3. The problem (1) cannot have more than one generalized solution in $V\left(Q_{T}\right)$.

Proof. Suppose that $\exists_{u_{1}, u_{2}} \in V\left(Q_{T}\right)$ two different generalized solutions for the problem (1). Hence, the difference $U=$ $u_{1}-u_{2}$ solves

$$
\left\{\begin{array}{l}
a U_{t t t}+\beta U_{t t}-c^{2} \Delta U-b \Delta U_{t}-\int_{0}^{t} h(t-s) \Delta U(s) d s=0 \\
U(x, 0)=U_{t}(x, 0)=U_{t t}(x, 0)=0 \\
\frac{\partial U}{\partial \eta}=\int_{0}^{t} \int_{\Omega} U(\xi, \tau) d \xi d \tau, x \in \partial \Omega
\end{array}\right.
$$


and (9) gives

$$
\begin{aligned}
a\left(U_{t t},\right. & \left.v_{t}\right)_{L^{2}\left(Q_{T}\right)}+\beta\left(U_{t}, v_{t}\right)_{L^{2}\left(Q_{T}\right)}+c^{2}(\nabla U, \nabla v)_{L^{2}\left(Q_{T}\right)} \\
& +b\left(\nabla U_{t}, \nabla v\right)_{L^{2}\left(Q_{T}\right)}+\left(\nabla W_{t}, \nabla v\right)_{L^{2}\left(Q_{T}\right)} \\
= & -c^{2} \int_{0}^{T} \int_{\partial \Omega} v\left(\int_{0}^{t} \int_{\Omega} U(\xi, \tau) d \xi d \tau\right) d s_{x} d t \\
& -b \int_{0}^{T} \int_{\partial \Omega} v \int_{\Omega} U(\xi, \tau) d \xi d s_{x} d t \\
& -\int_{0}^{T} \int_{\partial \Omega} v_{t}\left(\int_{\Omega} W(\xi, \tau) d \xi\right) d s_{x} d t .
\end{aligned}
$$

where

$$
W(x, t)=\int_{0}^{t} h(t-\sigma) U(x, \sigma) d \sigma .
$$

Let the function

$$
v(x, t)= \begin{cases}\int_{t}^{\tau} U(x, s) d s & 0 \leq t \leq \tau, \\ 0, & \tau \leq t \leq T .\end{cases}
$$

It is obvious that $v \in W\left(Q_{T}\right)$ and $v_{t}(x, t)=-U(x, t)$ for all $t \in[0, \tau]$. By integration by parts in the LHS of (75) that yields

$$
\begin{aligned}
-a\left(U_{t t}, v_{t}\right)_{L^{2}\left(Q_{T}\right)}= & a\left(U_{\tau}(x, \tau), U(x, \tau)\right)_{L^{2}(\Omega)} \\
& -a \int_{0}^{\tau}\left\|U_{t}(x, t)\right\|_{L^{2}(\Omega)}^{2} d t, \\
-\beta\left(U_{t}, v_{t}\right)_{L^{2}\left(Q_{T}\right)}= & \frac{\beta}{2}\|U(x, \tau)\|_{L^{2}(\Omega)}^{2}, \\
c^{2}(\nabla U, \nabla v)_{L^{2}\left(Q_{T}\right)}= & \frac{c^{2}}{2}\|\nabla v(x, 0)\|_{L^{2}(\Omega)}^{2}, \\
(\nabla W, \nabla v)_{L^{2}\left(Q_{T}\right)} \leq & h_{0} \int_{0}^{\tau}\|\nabla v(x, t)\|_{L^{2}(\Omega)}^{2} d t \\
& +\frac{h_{0}}{2} \int_{0}^{\tau}\|\nabla U(x, t)\|_{L^{2}(\Omega)}^{2} d t \\
& +\frac{h_{0}}{2} \int_{0}^{\tau} h_{0} \nabla U(t) d t, \\
b\left(\nabla U_{t}, \nabla v\right)_{L^{2}\left(Q_{T}\right)} & b \int_{0}^{\tau}\left\|\nabla v_{t}(x, t)\right\|_{L^{2}(\Omega)}^{2} d t .
\end{aligned}
$$

Plugging (78)-(82) into (75), we obtain

$$
\begin{aligned}
& a\left(U_{\tau}(x, \tau), U(x, \tau)\right)_{L^{2}(\Omega)}+\frac{\beta}{2}\|U(x, \tau)\|_{L^{2}(\Omega)}^{2}+\frac{c^{2}}{2}\|\nabla v(x, 0)\|_{L^{2}(\Omega)}^{2} \\
& =a \int_{0}^{\tau}\left\|U_{\tau}(x, t)\right\|_{L^{2}(\Omega)}^{2} d t-b \int_{0}^{\tau}\left\|v_{t}(x, t)\right\|_{L^{2}(\Omega)}^{2} d t h_{0} \\
& \quad \cdot \int_{0}^{\tau}\|\nabla v(x, t)\|_{L^{2}(\Omega)}^{2} d t+\frac{h_{0}}{2} \int_{0}^{\tau}\|\nabla U(x, t)\|_{L^{2}(\Omega)}^{2} d t \\
& \quad+\frac{h_{0}}{2} h_{0} \nabla U(t) d t-\int_{0}^{T} \int_{\partial \Omega} v\left(\int_{0}^{t} \int_{\Omega} W(\xi, \tau) d \xi d \tau\right) d s_{x} d t \\
& \quad-c^{2} \int_{0}^{T} \int_{\partial \Omega} v\left(\int_{0}^{t} \int_{\Omega} U(\xi, \tau) d \xi d \tau\right) d s_{x} d t \\
& \quad-b \int_{0}^{T} \int_{\partial \Omega} v \int_{\Omega} U(\xi, \tau) d \xi d s_{x} d t .
\end{aligned}
$$

Now since

$$
v^{2}(x, t)=\left(\int_{t}^{\tau} U(x, s) d s\right)^{2} \leq \tau \int_{0}^{\tau} U^{2}(x, s) d s,
$$

then

$$
\|v\|_{L^{2}\left(Q_{T}\right)}^{2} \leq \tau^{2}\|U\|_{L^{2}\left(Q_{T}\right)}^{2} \leq T^{2}\|U\|_{L^{2}\left(Q_{T}\right)}^{2} .
$$

Applying the inequality of the trace, the RHS of (83) gives

$$
\begin{gathered}
c^{2} \int_{0}^{T} \int_{\partial \Omega} v\left(\int_{0}^{t} \int_{\Omega} U(\xi, \tau) d \xi d \tau\right) d s_{x} d t \\
\leq \frac{c^{2}}{2} T^{2}\{l(\varepsilon)+|\Omega||\partial \Omega|\} \int_{0}^{\tau}\|U(x, t)\|_{L^{2}(\Omega)}^{2} d t \\
\quad+\frac{c^{2}}{2} \varepsilon \int_{0}^{\tau}\|\nabla v(x, t)\|_{L^{2}(\Omega)}^{2} d t \\
b \int_{0}^{T} \int_{\partial \Omega} v \int_{\Omega} U(\xi, t) d \xi d s_{x} d t \\
\leq \frac{b}{2}\left\{T^{2} l(\varepsilon)+|\Omega||\partial \Omega|\right\} \int_{0}^{\tau}\|U(x, t)\|_{L^{2}(\Omega)}^{2} d t \\
\quad+\frac{b}{2} \varepsilon \int_{0}^{\tau}\|\nabla v(x, t)\|_{L^{2}(\Omega)}^{2} d t \\
\int_{0}^{T} \int_{\partial \Omega} \quad\left(\int_{0}^{t} \int_{\Omega} W(\xi, \tau) d \xi d \tau\right) d s_{x} d t \\
\leq \frac{1}{2} T^{2}\{l(\varepsilon)+|\Omega||\partial \Omega|\} \int_{0}^{\tau} h \circ U(t) d t \\
\quad+\frac{1}{2} T^{2} h \circ\{l(\varepsilon)+|\Omega||\partial \Omega|\} \int_{0}^{\tau}\|U(x, t)\|_{L^{2}(\Omega)}^{2} d t \\
\quad+\varepsilon \int_{0}^{\tau}\|\nabla v(x, t)\|_{L^{2}(\Omega)}^{2} d t .
\end{gathered}
$$



get

Combining the relations (86)-(83) and (87)-(88), we

$$
\begin{aligned}
a\left(U_{\tau}(x, \tau), U(x, \tau)\right)_{L^{2}(\Omega)}+\frac{\beta}{2}\|U(x, \tau)\|_{L^{2}(\Omega)}^{2} & \\
& +\frac{c^{2}}{2}\|\nabla v(x, 0)\|_{L^{2}(\Omega)}^{2} \\
\leq & \left\{\frac{c^{2}}{2} T^{2}(l(\varepsilon)+|\Omega \| \partial \Omega|)+\frac{\left(b+h_{0}\right)}{2}\left(T^{2} l(\varepsilon)+|\Omega \| \partial \Omega|\right)\right\} \\
& \cdot \int_{0}^{\tau}\|U(x, t)\|_{L^{2}(\Omega)}^{2} d t+\left(\left(\frac{c^{2}+b}{2}+1\right) \varepsilon+h(0)\right) \\
& \cdot \int_{0}^{\tau}\|\nabla v(x, t)\|_{L^{2}(\Omega)}^{2} d t+a \int_{0}^{\tau}\left\|U_{t}(x, t)\right\|_{L^{2}(\Omega)}^{2} d t \\
& +\frac{h_{0}}{2} \int_{0}^{\tau}\|\nabla U(x, t)\|_{L^{2}(\Omega)}^{2} d t+\frac{h_{0}}{2} \int_{0}^{\tau} h \circ \nabla U(t) d t \\
& +\frac{1}{2} T^{2}(l(\varepsilon)+|\Omega \| \partial \Omega|) \int_{0}^{\tau} h \circ U(t) d t .
\end{aligned}
$$

Next, multiplying (74) by $U_{t t}$ and integrating the result over $Q_{\tau}=\Omega \times(0, \tau)$, we find

$$
\begin{gathered}
a\left(U_{t t t}, U_{t t}\right)_{L^{2}\left(Q_{T}\right)}+\beta\left(U_{t t}, U_{t t}\right)_{L^{2}\left(Q_{T}\right)}-c^{2}\left(\Delta U, U_{t t}\right)_{L^{2}\left(Q_{T}\right)} \\
-b\left(\Delta U_{t}, U_{t t}\right)_{L^{2}\left(Q_{T}\right)}-\left(\Delta W, U_{t t}\right)_{L^{2}\left(Q_{T}\right)}=0 .
\end{gathered}
$$

An integration by parts in (91) yields

$$
\begin{gathered}
a\left(U_{t t t}, U_{t t}\right)_{L^{2}\left(Q_{T}\right)}=\frac{a}{2}\left\|U_{\tau \tau}(x, \tau)\right\|_{L^{2}(\Omega)}^{2}, \\
\beta\left(U_{t t}, U_{t t}\right)_{L^{2}\left(Q_{T}\right)}=\beta \int_{0}^{\tau}\left\|U_{t t}(x, t)\right\|_{L^{2}(\Omega)}^{2} d t \\
-c^{2}\left(\Delta U, U_{t t}\right)_{L^{2}\left(Q_{T}\right)}=c^{2}\left(\nabla U_{\tau}(x, \tau), \nabla U(x, \tau)\right)_{L^{2}(\Omega)} \\
-c^{2} \int_{0}^{\tau}\left\|\nabla U_{t}(x, t)\right\|_{L^{2}(\Omega)}^{2} d t \\
-c^{2} \int_{\partial \Omega} U_{\tau}(x, \tau)\left(\int_{0}^{\tau} \int_{\Omega} U(\xi, \eta) d \xi d \eta\right) d s_{x} \\
+c^{2} \int_{\partial \Omega}^{\tau} \int_{0}^{\tau} U_{t}(x, t) \int_{\Omega} U(\xi, t) d \xi d t d s_{x},
\end{gathered}
$$

$$
\begin{aligned}
-b\left(\Delta U_{t}, U_{t t}\right)_{L^{2}\left(Q_{T}\right)}= & \frac{b}{2}\left\|\nabla U_{t}(x, \tau)\right\|_{L^{2}(\Omega)}^{2} \\
& -b \int_{\partial \Omega} U_{t}(x, \tau) \int_{\Omega} U(\xi, \tau) d \xi d s_{x} \\
& +b \int_{0}^{\tau} \int_{\partial \Omega} U_{t}(x, t) \int_{\Omega} U_{t}(\xi, t) d \xi d s_{x} d t,
\end{aligned}
$$

$$
\begin{aligned}
\left(\nabla W, \nabla U_{t t}\right)_{L^{2}\left(Q_{T}\right)}= & -\frac{1}{2} h^{\prime} \circ \nabla U(\tau)-\frac{1}{2} h(\tau)\|\nabla U(x, \tau)\|_{L^{2}\left(Q_{T}\right)}^{2} \\
& +2\left(\nabla W(\tau), \nabla U_{\tau}\right)_{L^{2}(\Omega)} \\
& -\frac{1}{2} \int_{0}^{\tau} h^{\prime}(t)\|\nabla U(x, t)\|_{L^{2}(\Omega)}^{2} d t \\
& +\frac{1}{2} \int_{0}^{\tau} h^{\prime \prime} \circ \nabla U(t) d t \\
& +\int_{\partial \Omega} U_{\tau}(x, \tau) \int_{0}^{\tau} \int_{\Omega} W(\xi, \sigma) d \xi d \sigma d s_{x} \\
& -\int_{0}^{\tau} \int_{\partial \Omega} U_{t}(x, t) \int_{\Omega} W(\xi, t) d \xi d s_{x} d t .
\end{aligned}
$$

Substitution (91)-(95) into (90), we get the equality

$$
\begin{aligned}
\frac{a}{2}\left\|U_{\tau \tau}(x, \tau)\right\|_{L^{2}(\Omega)}^{2}+c^{2}\left(\nabla U_{\tau}(x, \tau), \nabla U(x, \tau)\right)_{L^{2}(\Omega)} \\
+\frac{b}{2}\left\|\nabla U_{\tau}(x, \tau)\right\|_{L^{2}(\Omega)}^{2}-\frac{1}{2} h^{\prime} \circ \nabla U(\tau) \\
\quad-\frac{1}{2} h(\tau)\|\nabla U(x, \tau)\|_{L^{2}(\Omega)}^{2}+2\left(\nabla W(\tau), \nabla U_{\tau}\right)_{L^{2}(\Omega)} \\
=-\beta \int_{0}^{\tau}\left\|U_{t t}(x, t)\right\|_{L^{2}(\Omega)}^{2} d t+c^{2} \int_{0}^{\tau}\left\|\nabla U_{t}(x, t)\right\|_{L^{2}(\Omega)}^{2} \\
+c^{2} \int_{\partial \Omega} U_{\tau}(x, \tau)\left(\int_{0}^{\tau} \int_{\Omega} U(\xi, t) d \xi d t\right) d s_{x} \\
\quad-c^{2} \int_{\partial \Omega}^{\tau} \int_{0}^{\tau} U_{t}(x, t) \int_{\Omega} U(\xi, t) d \xi d t d s_{x} \\
+b \int_{\partial \Omega} U_{\tau}(x, \tau) \int_{\Omega} U(\xi, \tau) d \xi d s_{x} \\
\quad-b \int_{0}^{\tau} \int_{\partial \Omega} U_{t}(x, t) \int_{\Omega} W(\xi, t) d \xi d s_{x} d t \\
+\int_{\partial \Omega} U_{\tau}(x, \tau) \int_{0}^{\tau} \int_{\Omega} W(\xi, \sigma) d \xi d \sigma d s_{x} \\
\quad-\int_{0}^{\tau} \int_{\partial \Omega}^{\tau} U_{t}(x, t) \int_{\Omega} W(\xi, t) d \xi d s_{x} d t \\
\quad+\frac{1}{2} \int_{0}^{\tau} h^{\prime}(t)\|\nabla U(x, t)\|_{L^{2}(\Omega)}^{2} d t \\
\quad-\frac{1}{2} \int_{0}^{\tau} h^{\prime \prime} \circ \nabla U(t) d t .
\end{aligned}
$$

The RHS of (96) can be bounded as follows

$$
\begin{aligned}
& c^{2} \int_{\partial \Omega} U_{\tau}(x, \tau)\left(\int_{0}^{T} \int_{\Omega} U(\xi, t) d \xi d t\right) d s_{x} \\
& \leq \frac{c^{2}}{2 \varepsilon_{1}^{\prime}}\left(\varepsilon\left\|\nabla U_{\tau}(\xi, \tau)\right\|_{L^{2}(\Omega)}^{2}+l(\varepsilon)\left\|U_{\tau}(x, \tau)\right\|_{L^{2}(\Omega)}^{2}\right) \\
& \quad+\frac{c^{2}}{2 \varepsilon_{1}^{\prime}} T\left|\partial \Omega\left\|\Omega \mid \int_{0}^{T}\right\| U(x, t) \|_{L^{2}(\Omega)}^{2} d t,\right.
\end{aligned}
$$




$$
\begin{aligned}
& -c^{2} \int_{\partial \Omega} \int_{0}^{\tau} U_{t}(x, t) \int_{\Omega} U(\xi, \tau) d \xi d t d s_{x} \\
& \leq \frac{c^{2}}{2} \int_{0}^{T}\left\{\varepsilon\left\|\nabla U_{t}(x, t)\right\|_{L^{2}(\Omega)}^{2}+l(\varepsilon)\left\|U_{t}(x, t)\right\|_{L^{2}(\Omega)}^{2}\right\} d t \\
& +\frac{c^{2}}{2}|\Omega||\partial \Omega| \int_{0}^{\tau}\|U(x, t)\|_{L^{2}(\Omega)}^{2} d t, \\
& b \int_{\partial \Omega} U_{\tau}(x, \tau)\|\| \int_{\Omega} U(\xi, \tau) d \xi d s_{x} \\
& \leq \frac{b}{2 \varepsilon_{2}^{\prime}}\left(\varepsilon\left\|\nabla U_{\tau} x(\xi, \tau)\right\|_{L^{2}(\Omega)}^{2}+l(\varepsilon)\left\|U_{\tau}(x, \tau)\right\|_{L^{2}(\Omega)}^{2}\right) \\
& +\frac{b}{2} \varepsilon_{2}^{\prime} T\left|\Omega\|\partial \Omega \mid\| U(x, \tau) \|_{L^{2}(\Omega)}^{2},\right. \\
& -b \int_{0}^{\tau} \int_{\partial \Omega} U_{t}(x, t) \int_{\Omega} U(\xi, t) d \xi d s_{x} d t \\
& \leq \frac{b}{2}\{l(\varepsilon)+T|\Omega||\partial \Omega|\} \int_{0}^{\tau}\left\|U_{t}(x, t)\right\|_{L^{2}(\Omega)}^{2} d t \\
& +\frac{b}{2} \varepsilon \int_{0}^{\tau}\left\|\nabla U_{t}(x, t)\right\|_{L^{2}(\Omega)}^{2} d t, \\
& \int_{\partial \Omega} U_{\tau}(x, \tau)\left(\int_{0}^{\tau} \int_{\Omega} W(\xi, \tau) d \xi d t\right) d s_{x} \\
& \leq\left(\frac{1}{2 \varepsilon_{3}}+\frac{1}{2 \varepsilon_{4}}\right)\left(\varepsilon\left\|\nabla U_{\tau}(x, \tau)\right\|_{L^{2}(\Omega)}^{2}+l(\varepsilon)\left\|U_{\tau}(x, \tau)\right\|_{L^{2}(\Omega)}^{2}\right) \\
& +\frac{1}{2} \varepsilon_{3} T h_{0}|\partial \Omega||\Omega| \int_{0}^{\tau}\|U(x, t)\|_{L^{2}(\Omega)}^{2} d t \\
& +\frac{1}{2} \varepsilon_{4} T|\partial \Omega||\Omega| \int_{0}^{\tau} h \circ U(t) d t, \\
& -\int_{\partial \Omega} \int_{0}^{\tau} U_{t}(x, t) \int_{\Omega} W(\xi, t) d \xi d t d s_{x} \\
& \leq \int_{0}^{\tau}\left\{\varepsilon\left\|\nabla U_{t}(x, t)\right\|_{L^{2}(\Omega)}^{2}+l(\varepsilon)\left\|U_{t}(x, t)\right\|_{L^{2}(\Omega)}^{2}\right\} d t \\
& +\frac{1}{2} h_{0}|\Omega||\partial \Omega| \int_{0}^{\tau}\|U(x, t)\|_{L^{2}(\Omega)}^{2} d t \\
& +\frac{1}{2}|\Omega||\partial \Omega| \int_{0}^{\tau} h \circ U(t) d t,
\end{aligned}
$$

So, combining inequalities (97)-(102), we obtain

$$
\begin{aligned}
\frac{a}{2} \| U_{\tau \tau} & (x, \tau)\left\|_{L^{2}(\Omega)}^{2}-\frac{1}{2} h^{\prime} \circ \nabla U(\tau)-\frac{1}{2} h(\tau)\right\| \nabla U(x, \tau) \|_{L^{2}(\Omega)}^{2} \\
& +c^{2}\left(\nabla U_{\tau}(x, \tau), \nabla U(x, \tau)\right)_{L^{2}(\Omega)} \\
& +2\left(\nabla W(x, \tau), \nabla U_{\tau}(x, \tau)\right)_{L^{2}(\Omega)} \\
& +\left\{\frac{b}{2}-\frac{c^{2}}{2 \varepsilon_{1}^{\prime}} \varepsilon-\frac{b}{2 \varepsilon_{2}^{\prime}} \varepsilon-\left(\frac{1}{2 \varepsilon_{3}}+\frac{1}{2 \varepsilon_{4}}\right) \varepsilon\right\}\left\|\nabla U_{\tau}(x, \tau)\right\|_{L^{2}(\Omega)}^{2}
\end{aligned}
$$

$$
\begin{aligned}
& -\left\{\frac{c^{2} l(\varepsilon)}{2 \varepsilon_{1}^{\prime}}+\frac{b l(\varepsilon)}{2 \varepsilon_{2}^{\prime}}+\left(\frac{1}{2 \varepsilon_{3}}+\frac{1}{2 \varepsilon_{4}}\right) l(\varepsilon)\left\|U_{\tau}(x, \tau)\right\|_{L^{2}(\Omega)}^{2}\right. \\
& -\frac{b}{2} \varepsilon_{2}^{\prime} T|\Omega||\partial \Omega|\|U(x, \tau)\|_{L^{2}(\Omega)}^{2} \\
\leq & \beta \int_{0}^{\tau}\left\|U_{t t}(x, t)\right\|_{L^{2}(\Omega)}^{2} d t+\frac{1}{2} T|\Omega|\left(\varepsilon_{3}+1\right) \int_{0}^{\tau} h \circ U(t) d t \\
& +\frac{1}{2} \int_{0}^{\tau} h^{\prime \prime} \circ U(t) d t-\frac{1}{2} \int_{0}^{\tau} h^{\prime}(t)\left\|U_{\tau}(x, \tau)\right\|_{L^{2}(\Omega)}^{2} d t \\
& +\left\{c^{2}+\frac{c^{2}}{2} \varepsilon+\frac{b}{2} \varepsilon\right\} \int_{0}^{\tau}\left\|\nabla U_{t}(x, t)\right\|_{L^{2}(\Omega)}^{2} d t \\
& +\left\{\frac { c ^ { 2 } } { 2 } \varepsilon _ { 1 } ^ { \prime } T \left|\Omega\left\||\partial \Omega|+\frac{1}{2}\left(c^{2}+h_{0}+T h_{0} \varepsilon_{4}\right)|\Omega \| \partial \Omega|\right\}\right.\right. \\
& \cdot \int_{0}^{\tau}\|U(x, t)\|_{L^{2}(\Omega)}^{2} d t \\
& +\left(\frac{b}{2}\{l(\varepsilon)+T|\Omega \| \partial \Omega|\}+\frac{1}{2}\left(c^{2}+1\right) l(\varepsilon)\right) \\
& \cdot \int_{0}^{\tau}\left\|U_{t}(x, t)\right\|_{L^{2}(\Omega)}^{2} d t .
\end{aligned}
$$

Adding side to side (89) and (103) that gives

$$
\begin{aligned}
a\left(U_{\tau}(x, \tau), U(x, \tau)\right)_{L^{2}(\Omega)} & +\left\{\frac{\beta}{2}-\frac{b}{2} \varepsilon_{2}^{\prime} T|\Omega||\partial \Omega|\right\}\|U(x, \tau)\|_{L^{2}(\Omega)}^{2} \\
& +\frac{c^{2}}{2}\|\nabla v(x, 0)\|_{L^{2}(\Omega)}^{2}+\frac{a}{2}\left\|U_{\tau \tau}(x, \tau)\right\|_{L^{2}(\Omega)}^{2} \\
& +c^{2}\left(\nabla U_{\tau}(x, \tau), \nabla U(x, \tau)\right)_{L^{2}(\Omega)} \\
& +\left\{\frac{b}{2}-\frac{c^{2}}{2 \varepsilon_{1}^{\prime}} \varepsilon-\frac{b}{2 \varepsilon_{2}^{\prime}} \varepsilon-\left(\frac{1}{2 \varepsilon_{3}}+\frac{1}{2 \varepsilon_{4}}\right) \varepsilon\right\} \\
& +\left\|\nabla U_{\tau}(x, \tau)\right\|_{L^{2}(\Omega)}^{2}-\left\{\frac{c^{2}}{2 \varepsilon_{1}^{\prime}}+\frac{b}{2 \varepsilon_{2}^{\prime}}+\left(\frac{1}{2 \varepsilon_{3}}+\frac{1}{2 \varepsilon_{4}}\right) l(\varepsilon)\right\} \\
& +l(\varepsilon)\left\|U_{\tau}(x, \tau)\right\|_{L^{2}(\Omega)}^{2}-\frac{1}{2} h^{\prime} \circ \nabla U(\tau) \\
+ & 2\left(\nabla W(x, \tau), \nabla U_{\tau}(x, \tau)\right)_{L^{2}(\Omega)} \\
\leq & \gamma_{4} \int_{0}^{\tau}\|U(x, t)\|_{L^{2}(\Omega)}^{2} d t+\left\{a+\frac{b}{2}(l(\varepsilon)+T|\Omega \| \partial \Omega|)\right. \\
& \left.+\left(\frac{c^{2}}{2}+1\right) l(\varepsilon)\right\} \int_{0}^{\tau}\left\|U_{t}(x, t)\right\|_{L^{2}(\Omega)}^{2} d t \\
& +\left(\left(\frac{c^{2}+b}{2}+1\right) \varepsilon+h_{0}\right) \int_{0}^{\tau}\|\nabla v(x, t)\|_{L^{2}(\Omega)}^{2} d t \\
+ & \left\{c^{2}+\frac{c^{2}}{2} \varepsilon+\frac{b}{2} \varepsilon+\frac{h_{0}}{4}\right\} \int_{0}^{\tau}\left\|\nabla U_{t}(x, t)\right\|_{L^{2}(\Omega)}^{2} d t^{2} \\
+ & \left(\frac{1}{2}|\Omega \| \partial \Omega|\left(\varepsilon_{3} T+1+T^{2}\right)+\frac{1}{2} T^{2} l(\varepsilon)\right) \int_{0}^{\tau} h \circ U(t) d t \\
+ & \frac{1}{2} \int_{0}^{\tau} h^{\prime \prime} \circ \nabla U(t) d t+\frac{h_{0}}{2} \int_{0}^{\tau} h \circ \nabla U(t) d t \\
+ & \left(\frac{h(0)}{4}+\frac{h_{0}}{2}\right) \int_{0}^{\tau}\|\nabla U(x, t)\|_{L^{2}(\Omega)}^{2} d t, \\
& =(104)
\end{aligned}
$$


where

$$
\begin{aligned}
\gamma_{4}:= & \left\{\frac{c^{2}}{2} T^{2}(l(\varepsilon)+|\Omega||\partial \Omega|)+\frac{b}{2}\left(T^{2} l(\varepsilon)+|\Omega||\partial \Omega|\right)\right. \\
& +\frac{1}{2} T^{2}\left(l(\varepsilon)+|\Omega||\partial \Omega| h_{0}\right)+\frac{c^{2}}{2 \varepsilon_{1}} \varepsilon_{1}^{\prime} T|\Omega||\partial \Omega| \\
& \left.+\frac{c^{2}}{2}|\Omega||\partial \Omega|+\frac{1}{2} h_{0}|\Omega||\partial \Omega|\left(\varepsilon_{4} T^{2}+1\right)\right\} .
\end{aligned}
$$

Now, the last term on the RHS of (104), we give the function $\theta(x, t)$ by

$$
\theta(x, t):=\int_{0}^{t} U(x, s) d s .
$$

Hence, we use (77), and we get

$$
\begin{aligned}
\mathrm{v}(\mathrm{x}, \mathrm{t}) & =\theta(\mathrm{x}, \tau)-\theta(\mathrm{x}, \mathrm{t}), \nabla \mathrm{v}(\mathrm{x}, 0)=\nabla \theta(\mathrm{x}, \tau), \\
\|\nabla v\|_{L^{2}\left(Q_{\tau}\right)}^{2} & =\|\nabla \theta(x, \tau)-\nabla \theta(x, t)\|_{L^{2}(\Omega)}^{2} \\
& \leq 2\left(\tau\|\nabla \theta(x, \tau)\|_{L^{2}(\Omega)}^{2}+\|\nabla \theta(x, t)\|_{L^{2}\left(Q_{\tau}\right)}^{2}\right) .
\end{aligned}
$$

And using the inequalities

$$
\begin{aligned}
& m_{1}^{\prime}\|U(x, \tau)\|_{L^{2}(\Omega)}^{2} \\
& \quad \leq m_{1}^{\prime}\|U(x, t)\|_{L^{2}\left(Q_{\tau}\right)}^{2}+m_{1}\left\|U_{t}(x, t)\right\|_{L^{2}\left(Q_{\tau}\right)}^{2}, \\
& m_{2}^{\prime}\left\|U_{\tau}(x, \tau)\right\|_{L^{2}(\Omega)}^{2} \\
& \quad \leq m_{2}^{\prime}\left\|U_{t}(x, t)\right\|_{L^{2}\left(Q_{\tau}\right)}^{2}+m_{2}\left\|U_{t t}(x, t)\right\|_{L^{2}\left(Q_{\tau}\right)}^{2}, \\
& m_{3}^{\prime}\|\nabla U(x, \tau)\|_{L^{2}(\Omega)}^{2} \\
& \quad \leq m_{3}^{\prime}\|\nabla U(x, t)\|_{L^{2}\left(Q_{\tau}\right)}^{2}+m_{3}\left\|\nabla U_{t}(x, t)\right\|_{L^{2}\left(Q_{\tau}\right)}^{2}, \\
& m_{4}^{\prime} h \circ U(\tau) \\
& \quad \leq m_{4}^{\prime}\left(1+\frac{\zeta}{2}\right) \int_{0}^{\tau} h^{\prime} \circ U(t) d t+m_{4}\left\|\nabla U_{t}(x, \tau)\right\|_{L^{2}\left(Q_{\tau}\right)}^{2}, \\
& -\frac{a}{2}\left\|U_{\tau}(x, \tau)\right\|_{L^{2}(\Omega)}^{2}-\frac{a}{2}\|U(x, \tau)\|_{L^{2}(\Omega)}^{2} \\
& \quad \leq a\left(U_{\tau}(x, \tau), U(x, \tau)\right)_{L^{2}(\Omega)} \\
& -\frac{c^{2}}{2 \varepsilon_{3}^{\prime}}\left\|\nabla U_{\tau}(x, \tau)\right\|_{L^{2}(\Omega)}^{2}-\frac{c^{2}}{2} \varepsilon_{3}^{\prime}\|\nabla U(x, \tau)\|_{L^{2}(\Omega)}^{2} \\
& \quad \leq c^{2}\left(\nabla U_{\tau}(x, \tau), \nabla U(x, \tau)\right)_{L^{2}(\Omega)} \\
& -\left(\frac{1}{4 \varepsilon_{9}}+\frac{h_{0}}{4 \varepsilon_{8}}\right)\left\|\nabla U_{\tau}(x, \tau)\right\|_{L^{2}(\Omega)}^{2} \\
& \quad-h_{0} \varepsilon_{8}\|\nabla U(x, \tau)\|_{L^{2}(\Omega)}^{2}-\varepsilon_{9} h_{0} \nabla U(\tau) \\
& \quad \leq 2\left(\nabla W(x, \tau), \nabla U_{\tau}(x, \tau)\right)_{L^{2}(\Omega)} .
\end{aligned}
$$

Let

$$
\begin{aligned}
& \left\{\begin{array}{l}
m_{1}^{\prime}:=\frac{a}{2}+\frac{b}{2} \varepsilon_{2}^{\prime} T|\Omega||\partial \Omega|, \\
m_{2}^{\prime}:=1+\frac{a}{2}-\frac{c^{2}}{2 \varepsilon_{3}^{\prime}}-\left\{\frac{c^{2}}{2 \varepsilon_{1}^{\prime}}+\frac{b}{2 \varepsilon_{2}^{\prime}}+\frac{1}{2 \varepsilon_{3}}+\frac{1}{2 \varepsilon_{4}}\right\} l(\varepsilon),
\end{array}\right. \\
& m_{3}^{\prime}:=\frac{c^{2}}{2} \varepsilon_{3}^{\prime}+h_{0} \varepsilon_{8},
\end{aligned}
$$

and we choose $\varepsilon_{1}^{\prime}, \varepsilon_{2}^{\prime}, \varepsilon_{3}^{\prime}, \varepsilon_{8}$ and $\varepsilon_{9}$ sufficiently large

$$
\frac{c^{2}}{2 \varepsilon_{1}^{\prime}} \varepsilon+\frac{b}{2 \varepsilon_{2}^{\prime}} \varepsilon+\frac{c^{2}}{2 \varepsilon_{3}^{\prime}}+\left(\frac{1}{4 \varepsilon_{9}}+\frac{h_{0}}{4 \varepsilon_{9}}\right) \varepsilon<\frac{b}{2} .
$$

As $\tau$ is arbitrary, and we get

$$
A:=\frac{c^{2}}{2}-2 \tau \varepsilon\left(\left(c^{2}+b\right) / 2+1+h_{0}\right)>0 .
$$

Thus, inequality (104) takes the form

$$
\begin{aligned}
\frac{\beta}{2} \| & U(x, \tau)\left\|_{L^{2}(\Omega)}^{2}+\right\| U_{\tau}(x, \tau)\left\|_{L^{2}(\Omega)}^{2}+\right\| \nabla U(x, \tau) \|_{L^{2}(\Omega)}^{2} \\
& +\{\underbrace{\frac{b}{2}-\frac{c^{2}}{2 \varepsilon_{1}^{\prime}} \varepsilon-\frac{b}{2 \varepsilon_{2}^{\prime}} \varepsilon-\frac{c^{2}}{2 \varepsilon_{3}^{\prime}}+\frac{1}{4 \varepsilon_{9}}+\frac{h_{0}}{4 \varepsilon_{8}}}_{\gamma_{6}}\} \\
& +\left\|\nabla U_{\tau}(x, \tau)\right\|_{L^{2}(\Omega)}^{2}-\frac{1}{2} h^{\prime} \circ \nabla U(\tau)-h^{\prime} \circ U(\tau) \\
& +\frac{a}{2}\left\|U_{\tau \tau}(x, \tau)\right\|_{L^{2}(\Omega)}^{2}+A\|\nabla \theta(x, \tau)\|_{L^{2}(\Omega)}^{2} \\
\leq & \left.\gamma_{4}+m_{1}^{\prime}\right) \int_{0}^{\tau}\|U(x, t)\|_{L^{2}(\Omega)}^{2} d t+\gamma_{5} \int_{0}^{\tau}\left\|U_{t}(x, t)\right\|_{L^{2}(\Omega)}^{2} d t \\
+ & \left(m_{3}^{\prime}+\frac{h_{0}}{4}\right) \int_{0}^{\tau}\|\nabla U(x, t)\|_{L^{2}(\Omega)}^{2} d t \\
+ & \left(\beta+m_{2}^{\prime}\right) \int_{0}^{\tau}\left\|U_{t t}(x, t)\right\|_{L^{2}(\Omega)}^{2} d t \\
& \left.+\int_{\gamma_{7}}^{c^{2}+\frac{c^{2}}{2} \varepsilon+\frac{b}{2} \varepsilon+\frac{h_{0}}{4}+\frac{h(0)}{2}+m_{3}^{\prime}}\right\} \\
& +\underbrace{\tau}_{0}\|\nabla \theta(x, t)\|_{L^{2}(\Omega)}^{2} d t-\frac{1}{2} \int_{0}^{\tau} h^{\prime \prime} \circ \nabla U(t) d t \\
& (\underbrace{\tau}_{t}+1) \int_{0}^{\tau} h^{\prime} \circ \nabla U(t) d t
\end{aligned}
$$




$$
\begin{aligned}
& \underbrace{-\frac{1}{\zeta}\left(\frac{1}{2}|\Omega||\partial \Omega|\left(\varepsilon_{3} T+1+T^{2}\right)+\frac{1}{2} T^{2} l(\varepsilon)+1\right)}_{\gamma_{9}} \\
& \cdot \int_{0}^{\tau} h^{\prime} \circ U(t) d t,
\end{aligned}
$$

where

$$
\begin{aligned}
\gamma_{5}:= & a+\frac{b}{2}(l(\varepsilon)+T|\Omega||\partial \Omega|)+\left(\frac{c^{2}}{2}+1\right) l(\varepsilon)+\frac{h(0)}{2} \\
& +m_{1}^{\prime}+m_{2}^{\prime} .
\end{aligned}
$$

$$
D^{\prime}:=\frac{\max \left\{(\beta / 2), 1,\left(\eta^{2} / 2\right), \gamma_{6},(a / 2), A\right\}}{\min \left\{\left(\gamma_{4}+m_{1}^{\prime}\right), \gamma_{5},\left(m_{3}^{\prime}+\left(h_{0} / 4\right)\right), \gamma_{7},\left(\beta+m_{2}^{\prime}\right),\left(\left(\varepsilon_{9} / \zeta\right)+1\right), \gamma_{8}, \gamma_{9}\right\}} .
$$

We get

$$
\begin{aligned}
& \|U(x, \tau)\|_{L^{2}(\Omega)}^{2}+\left\|U_{\tau}(x, \tau)\right\|_{L^{2}(\Omega)}^{2}+\|\nabla U(x, \tau)\|_{L^{2}(\Omega)}^{2} \\
& \quad-h^{\prime} \circ U(\tau)+\left\|\nabla U_{\tau}(x, \tau)\right\|_{L^{2}(\Omega)}^{2}+\left\|U_{\tau \tau}(x, \tau)\right\|_{L^{2}(\Omega)}^{2} \\
& \quad+\|\nabla \theta(x, \tau)\|_{L^{2}(\Omega)}^{2}-h^{\prime} \circ \nabla U(\tau) \\
& \leq D^{\prime} \int_{0}^{\tau}\left\{\|U(x, t)\|_{L^{2}(\Omega)}^{2}+\left\|U_{t}(x, t)\right\|_{L^{2}(\Omega)}^{2}\right. \\
& \quad+\|\nabla U(x, t)\|_{L^{2}(\Omega)}^{2}-h^{\prime} \circ U(t)+\left\|\nabla U_{t}(x, t)\right\|_{L^{2}(\Omega)}^{2} \\
& \left.\quad+\left\|U_{t t}(x, t)\right\|_{L^{2}(\Omega)}^{2}+\|\nabla \theta(x, t)\|_{L^{2}(\Omega)}^{2}-h^{\prime} \circ \nabla U(t)\right\} d t
\end{aligned}
$$

where
Hence, applying Gronwall's lemma to (114) gives

$$
\begin{aligned}
& \|U(x, \tau)\|_{L^{2}(\Omega)}^{2}+\left\|U_{\tau}(x, \tau)\right\|_{L^{2}(\Omega)}^{2}+\left\|\nabla U_{\tau}(x, \tau)\right\|_{L^{2}(\Omega)}^{2} \\
& \quad-h^{\prime} \circ U(\tau)+\left\|U_{\tau \tau}(x, \tau)\right\|_{L^{2}(\Omega)}^{2}+\|\nabla U(x, \tau)\|_{L^{2}(\Omega)}^{2} \\
& \quad+\|\nabla \theta(x, \tau)\|_{L^{2}(\Omega)}^{2}-h^{\prime} \circ \nabla U(\tau) \leq 0
\end{aligned}
$$

for all $\tau \in\left[0,\left(c^{2} / 4 \varepsilon\left(\left(\left(c^{2}+b\right) / 2\right)+1+h_{0}\right)\right)\right]$.

For the intervals, we use the same method

$$
\tau \in\left[\frac{(m-1) c^{2}}{4 \varepsilon\left(\left(\left(c^{2}+b\right) / 2\right)+1+h_{0}\right)}, \frac{m c^{2}}{4 \varepsilon\left(\left(\left(c^{2}+b\right) / 2\right)+1+h_{0}\right)}\right]
$$

to cover the whole interval $[0, T]$ and thus proving that $U(x, \tau)=0, \forall \tau$ in $[0, T]$. Hence, the uniqueness is proved.

\section{Conclusion}

The objective of this work is the study of solvability of the Moore-Gibson-Thompson equation with viscoelastic memory term and integral condition by using the Galerkin method. The MGT equation is a nonlinear partial differential equation that arises in hydrodynamics and some physical applications. Recent developments in numerical schemes for solving MGT have placed immense interest in nonlinear dispersive wave models. In the next work, we will try to use the same method with Boussinesque and Hall-MHD equations which are nonlinear partial differential equation that arises in hydrodynamics and some physical applications. It was subsequently applied to problems in the percolation of water in porous subsurface strata (see $[6,15-24]$, for example, $[10,11,25,26])$ by using some famous algorithms (see [27-29]).

\section{Data Availability}

No data were used to support the study.

\section{Conflicts of Interest}

The authors declare that they have no conflicts of interest.

\section{Acknowledgments}

The fifth author extends his appreciation to the Deanship of Scientific Research at King Khalid University for funding this work through research groups program under grant (R.G.P-2/1/42).

\section{References}

[1] S. Boulaaras, A. Zaraï, and A. Draifia, "Galerkin method for nonlocal mixed boundary value problem for the MooreGibson-Thompson equation with integral condition," Mathematical Methods in the Applied Sciences, vol. 42, no. 8, pp. 2664-2679, 2019.

[2] I. Lasiecka and X. Wang, "Moore-Gibson-Thompson equation with memory, part I: exponential decay of energy," Zeitschrift für Angewandte Mathematik und Physik, vol. 67, no. 2, 2016.

[3] G. Lebon and A. Cloot, "Propagation of ultrasonic sound waves in dissipative dilute gases and extended irreversible thermodynamics," Wave Motion, vol. 11, no. 1, pp. 23-32, 1989.

[4] R. Marchand, T. McDevitt, and R. Triggiani, "An abstract semigroup approach to the third-order Moore-GibsonThompson partial differential equation arising in high- 
intensity ultrasound: structural decomposition. spectral analysis, exponential stability," Mathematical Methods in the Applied Sciences, vol. 35, no. 15, pp. 1896-1929, 2012.

[5] K. Naugolnykh and L. Ostrovsky, Nonlinear wave processes in acoustics, Cambridge University Press, Cambridge, 1998.

[6] L. S. Pulkina, "A nonlocal problem with integral conditions for hyperbolic equations," Electronic Journal of Differential Equations, vol. 45, pp. 1-6, 1999.

[7] P. Thompson, A: Compressible-Fluid Dynamics, McGrawHill, New York, 1972.

[8] P. Stokes, "XXXVIII. An examination of the possible effect of the radiation of heat on the propagation of sound," The London, Edinburgh, and Dublin Philosophical Magazine and Journal of Science, vol. 1, no. 4, pp. 305-317, 1851.

[9] S. Mesloub, "A nonlinear nonlocal mixed problem for a second order pseudoparabolic equation," Journal of Mathematical Analysis and Applications, vol. 316, no. 1, pp. 189-209, 2006.

[10] R. P. Agarwal, S. Gala, and M. A. Ragusa, "A regularity criterion in weak spaces to Boussinesq equations," Mathematics, vol. 8, no. 6, p. 920, 2020.

[11] R. P. Agarwal, S. Gala, and M. A. Ragusa, "A regularity criterion of the 3D MHD equations involving one velocity and one current density component in Lorentz space," Zeitschrift für Angewandte Mathematik und Physik, vol. 71, no. 3, 2020.

[12] S. Boulaaras, "Solvability of the Moore-Gibson-Thompson equation with viscoelastic memory term and integral condition via Galerkin Method," Fractals, vol. 29, no. 5, article 2140021, 2021.

[13] B. Kaltenbacher, I. Lasiecka, and R. Marchand, "Well-posedness and exponential decay rates for the Moore-GibsonThompson equation arising in high intensity ultrasound," Control and Cybernetics, vol. 40, no. 4, pp. 1245-1264, 2011.

[14] I. Lasiecka and X. Wang, "Moore-Gibson-Thompson equation with memory, part II: general decay of energy," Journal of Differential Equations, vol. 259, no. 12, pp. 7610-7635, 2015.

[15] O. A. Ladyzhenskaya, The boundary value problems of mathematical physics, Springer-Verlag, New York Heidelberg Tokyo, 1985.

[16] D. M. Cahlon, D. M. Kulkarni, and P. Shi, "Stepwise stability for the heat equation with a nonlocal constraint," SIAM Journal on Numerical Analysis, vol. 32, no. 2, pp. 571-593, 1995.

[17] J. R. Cannon, "The solution of the heat equation subject to the specification of energy," Quarterly of Applied Mathematics, vol. 21, no. 2, pp. 155-160, 1963.

[18] V. Capasso and K. Kunisch, "A reaction-diffusion system arising in modelling man-environment diseases," Quarterly of Applied Mathematics, vol. 46, no. 3, pp. 431-450, 1988.

[19] Y.S. Choi and K. Y. Chan, "A parabolic equation with nonlocal boundary conditions arising from electrochemistry," Nonlinear Analysis, vol. 18, no. 4, pp. 317-331, 1992.

[20] R. E. Ewing and T. Lin, "A class of parameter estimation techniques for fluid flow in porous media," Advances in Water Resources, vol. 14, no. 2, pp. 89-97, 1991.

[21] P. M. Jordan, "Second-sound phenomena in inviscid, thermally relaxing gases," Discrete \& Continuous Dynamical Systems - B, vol. 19, no. 7, pp. 2189-2205, 2014.

[22] B. Kaltenbacher, I. Lasiecka, and M. Pospieszalska, "Wellposedness and exponential decay of the energy in the nonlinear Jordan-Moore-Gibson-Thompson equation arising in high intensity ultrasound," Mathematical Models and Methods in Applied Sciences, vol. 22, no. 11, pp. 195-207, 2012.

[23] S. Mesloub and F. Mesloub, "On the higher dimension Boussinesq equation with nonclassical condition," Mathematicsl Methods in the Applied Sciences, vol. 34, no. 5, pp. 578-586, 2011.

[24] A. M. Alghamdi, S. Gala, and M. A. Ragusa, "A regularity criterion for local strong solutions to the 3D Stokes-MHD equations," Annales Polonici Mathematici, vol. 124, no. 3, pp. 247-255, 2020.

[25] A. Barbagallo, S. Gala, M. A. Ragusa, and M. Théra, "On the regularity of weak solutions of the Boussinesq equations in Besov spaces," Vietnam Journal of Mathematics, 2020.

[26] R. P. Agarwal, A. M. A. Alghamdi, S. Gala, and M. A. Ragusa, "On the continuation principle of local smooth solution for the Hall-MHD equations," Applicable Analysis, pp. 1-9, 2020.

[27] M. H. Boulaaras and M. Haiour, " $L^{\infty}$-asymptotic behavior for a finite element approximation in parabolic quasi-variational inequalities related to impulse control problem," Applied Mathematics and Computation, vol. 217, no. 14, pp. 64436450, 2011.

[28] M. H. Boulaaras and M. Haiour, "A new proof for the existence and uniqueness of the discrete evolutionary HJB equations," Applied Mathematics and Computation, vol. 262, pp. 42-55, 2015.

[29] S. Boulaaras, "Some new properties of asynchronous algorithms of theta scheme combined with finite elements methods for an evolutionary implicit 2-sided obstacle problem," Mathematical Methods in the Applied Sciences, vol. 40, no. 18, pp. 7231-7239, 2017. 